\title{
OzDES multi-object fibre spectroscopy for the Dark Energy Survey: results and second data release
}

C. Lidman ${ }^{\odot},{ }^{1 \star}$ B. E. Tucker, ${ }^{1}$ T. M. Davis ${ }^{\oplus},{ }^{2}$ S. A. Uddin, ${ }^{3}$ J. Asorey, ${ }^{4}$ K. Bolejko ${ }^{\odot}, 5$

D. Brout,${ }^{6} \dagger$ J. Calcino, ${ }^{2}$ D. Carollo, ${ }^{7}$ A. Carr,${ }^{2}$ M. Childress ${ }^{\oplus},{ }^{8}$ J. K. Hoormann, ${ }^{2}$

R. J. Foley, ${ }^{9}$ L. Galbany ${ }^{\oplus},{ }^{10}$ K. Glazebrook, ${ }^{11}$ S. R. Hinton ${ }^{\odot}, 2$ R. Kessler, ${ }^{12,13}$

A. G. Kim ${ }^{\oplus},{ }^{14}$ A. King, ${ }^{15}$ A. Kremin, ${ }^{16}$ K. Kuehn, ${ }^{17,18}$ D. Lagattuta ${ }^{\odot}, 19,20$

G. F. Lewis ${ }^{\oplus},{ }^{21}$ E. Macaulay ${ }^{\oplus},{ }^{22}$ U. Malik, ${ }^{1}$ M. March ${ }^{\odot}, 6$ P. Martini, ${ }^{23,24}$ A. Möller, ${ }^{25}$

D. Mudd,${ }^{24}$ R. C. Nichol, ${ }^{22}$ F. Panther ${ }^{\oplus},{ }^{1,26}$ D. Parkinson ${ }^{\oplus},{ }^{27}$ M. Pursiainen ${ }^{\odot}, 8$

M. Sako, ${ }^{6}$ E. Swann, ${ }^{22}$ R. Scalzo, ${ }^{1,28}$ D. Scolnic, ${ }^{29}$ R. Sharp,${ }^{1}$ M. Smith ${ }^{\oplus}, 8$

N. E. Sommer, ${ }^{1}$ M. Sullivan, ${ }^{8}$ S. Webb,${ }^{11}$ P. Wiseman ${ }^{\oplus},{ }^{8}$ Z. Yu, ${ }^{24}$ F. Yuan, ${ }^{1}$

B. Zhang ${ }^{\oplus},{ }^{1}$ T. M. C. Abbott, ${ }^{30}$ M. Aguena, ${ }^{31,32}$ S. Allam, ${ }^{33}$ J. Annis, ${ }^{33}$ S. Avila ${ }^{\oplus}, 34$

E. Bertin, ${ }^{35,36}$ S. Bhargava, ${ }^{37}$ D. Brooks, ${ }^{38}$ A. Carnero Rosell ${ }^{\oplus},{ }^{4}$

M. Carrasco Kind ${ }^{\oplus}, 39,40$ J. Carretero, ${ }^{41}$ F. J. Castander, ${ }^{42,43}$ M. Costanzi, ${ }^{44,45}$

L. N. da Costa, ${ }^{32,46}$ J. De Vicente ${ }^{\oplus},{ }^{4}$ P. Doel, ${ }^{38}$ T. F. Eifler ${ }^{\oplus},{ }^{47,48}$ S. Everett, ${ }^{9}$

P. Fosalba, ${ }^{42,43}$ J. Frieman, ${ }^{33,13}$ J. García-Bellido, ${ }^{34}$ E. Gaztanaga ${ }^{\oplus}, 42,43$

D. Gruen ${ }^{\oplus}, 49,50,51$ R. A. Gruendl, ${ }^{39,40}$ J. Gschwend, ${ }^{32,46}$ G. Gutierrez, ${ }^{33}$

W. G. Hartley, ${ }^{52,38,53}$ D. L. Hollowood, ${ }^{9}$ K. Honscheid, ${ }^{23,54}$ D. J. James, ${ }^{55}$

N. Kuropatkin, ${ }^{33}$ T. S. Li ${ }^{\oplus}, 56,57$ M. Lima, ${ }^{31,32}$ H. Lin, ${ }^{33}$ M. A. G. Maia, ${ }^{32,46}$

J. L. Marshall, ${ }^{58}$ P. Melchior ${ }^{\oplus},{ }^{56}$ F. Menanteau, ${ }^{39,40}$ R. Miquel, ${ }^{59,41}$ A. Palmese ${ }^{\bullet}, 33,13$

F. Paz-Chinchón, ${ }^{39,40}$ A. A. Plazas ${ }^{\oplus},{ }^{56}$ A. Roodman, ${ }^{50,51}$ E. S. Rykoff,,${ }^{50,51}$ E. Sanchez, ${ }^{4}$

B. Santiago, ${ }^{60,32}$ V. Scarpine, ${ }^{33}$ M. Schubnell, ${ }^{16}$ S. Serrano, ${ }^{42,43}$ I. Sevilla-Noarbe, ${ }^{4}$

E. Suchyta ${ }^{\oplus},{ }^{61}$ M. E. C. Swanson, ${ }^{40}$ G. Tarle, ${ }^{16}$ D. L. Tucker, ${ }^{33}$ T. N. Varga, ${ }^{62,63}$

A. R. Walker, ${ }^{30}$ W. Wester, ${ }^{33}$ and R. D. Wilkinson ${ }^{37}$ (DES Collaboration)

Affiliations are listed at the end of the paper

Accepted 2020 May 4. Received 2020 April 22; in original form 2020 March 19

\begin{abstract}
We present a description of the Australian Dark Energy Survey (OzDES) and summarize the results from its 6 years of operations. Using the $2 \mathrm{dF}$ fibre positioner and AAOmega spectrograph on the 3.9-m Anglo-Australian Telescope, OzDES has monitored 771 active galactic nuclei, classified hundreds of supernovae, and obtained redshifts for thousands of galaxies that hosted a transient within the 10 deep fields of the Dark Energy Survey. We also present the second OzDES data release, containing the redshifts of almost 30000 sources, some as faint as $r_{\mathrm{AB}}=24 \mathrm{mag}$, and 375000 individual spectra. These data, in combination with the time-series photometry from the Dark Energy Survey, will be used to measure the expansion history of the Universe out to $z \sim 1.2$ and the masses of hundreds of black holes out to $z \sim 4$. OzDES is a template for future surveys that combine simultaneous monitoring of targets with wide-field imaging cameras and wide-field multi-object spectrographs.
\end{abstract}


Key words: transients: supernovae-quasars: supermassive black holes-cosmology: dark energy - surveys - catalogues - techniques: spectroscopic.

\section{INTRODUCTION}

Over the next 10 years, surveys using the next generation of multiobject spectroscopic facilities fed by rapidly configurable fibre positioning systems will obtain the spectra of tens of millions of sources. Facilities such as the Subaru Prime Focus Spectrograph (Tamura et al. 2018) on the 8.2-m Subaru telescope, the Dark Energy Spectroscopic Instrument (DESI; DESI Collaboration 2016; Vargas-Magana et al. 2019) on the 4-m Mayall telescope, and the 4-m Multi-Object Spectroscopic Telescope (4MOST; de Jong et al. 2019) on the 4-m VISTA telescope are either being built or on the cusp of entering operation. Other facilities, such as Mauna Kea Spectroscopic Explorer (MSE; The MSE Science Team et al. 2019) and MANIFEST (Lawrence et al. 2018) for the Giant Magellan Telescope, are being planned. Over the same time frame, the Legacy Survey of Space and Time (LSST; LSST Science Collaboration 2017) at the Rubin Observatory will start imaging the entire southern sky multiple times in multiple pass-bands over 10 years. The advent of the LSST in combination with these spectroscopic facilities will allow contemporaneous imaging and spectroscopic studies of tens of thousands of targets.

As a herald to these future surveys, the Australian Dark Energy Survey $(\mathrm{OzDES})^{1}$ has used the $2 \mathrm{dF}$ fibre positioner on the AngloAustralian Telescope (AAT) to obtain spectra of thousands of sources in the 10 deep fields of the Dark Energy Survey (DES) over the 6 years that DES ran. OzDES has two main scientific goals: (i) constraining the dark energy equation-of-state parameter using type Ia supernovae ( $\mathrm{SNe}$ Ia) and (ii) measuring the mass of supermassive black holes over a broad range of redshifts using active galactic nucleus (AGN) reverberation mapping (RM). Additionally, OzDES has obtained spectra for a number of ancillary projects, such as improving and quantifying the accuracy of redshifts obtained from broad-band photometry. In total, about 40 per cent of the fibre hours not used for active $\mathrm{SNe}$, host galaxies, and AGN were used for these projects.

This paper presents results from the full 6 years of OzDES, and describes the second OzDES data release. ${ }^{2}$ After giving a brief summary of the principal scientific aims of OzDES in Section 2, we provide an overview of OzDES operations for the last 3 years of the survey. Throughout the paper, we will use Y4, Y5, and Y6 to denote the fourth, fifth, and sixth years of OzDES, respectively. The observing strategy for the first 3 years of OzDES (Y1, Y2, and Y3) and the precursor survey are discussed in two papers: Yuan et al. (2015) give an overview up the end of Y1 (2013), including the OzDES precursor survey, and Childress et al. (2017) give an overview up until the end of Y3 (2015). We will refer to these papers as Yu15 and Ch17 henceforth. The reliability and completeness of OzDES redshifts are analysed in Section 4, and the data release is described in Section 5. In the sections that follow, we examine various aspects of the survey, such as the frequency at which we were able to target AGN, and how the signal-to-noise ratio behaves with time for objects that have the longest integration times: the host galaxies of transients. Before giving the summary of the paper in the final section, we compare OzDES to TiDES, an OzDES-like survey

\footnotetext{
${ }^{1}$ Australian (aka 'Oz') Dark Energy Survey.

${ }^{2}$ The first and second OzDES data releases are available from Data Central. Future releases will be announced on Data Central.
}

using the 4MOST facility (Swann et al. 2019). All magnitudes listed in this paper are measured using 2-arcsec diameter apertures and are on the $\mathrm{AB}$ magnitude system.

\section{DES AND OZDES}

DES was a 6-year programme, ending in 2019, using the DECam imager (Flaugher et al. 2015) on the CTIO Blanco 4-m telescope in Chile. DES consisted of two surveys: a wide-field survey covering 5000 square deg, and a deep survey consisting of 10 DECam fields located in several well-studied extragalactic fields. These 10 fields, the coordinates of which can be found in table 2 of D'Andrea et al. (2018), were targeted repeatedly in griz over the course of the first 5 years of DES with a cadence of approximately 6 days (Diehl et al. 2018), and consist of 8 relatively shallow fields and 2 extra deep fields (known as X3 and C3). The regular cadence facilitated the discovery and follow-up of transient sources, such as $\mathrm{SNe}$, and the monitoring of variable objects, such as AGN.

DES aims to place constraints on the dark energy equation-ofstate parameter and deviations from General Relativity using four astronomical probes: weak gravitational lensing, galaxy clusters, the large-scale distribution of galaxies, and type Ia SNe. Results from small subset of the DES data set have been published in a number of papers, the most recent of which are constraints that combine $\mathrm{SNe}$, gravitational lensing, and galaxy clustering (Dark Energy Survey 2019a).

The DES data have been used in many studies covering a diverse range of fields (Dark Energy Survey 2016). Notable examples include studies of bodies in the outer Solar system (for example, Khain et al. 2018), AGN accretion disc sizes (Mudd et al. 2018; Yu et al. 2018), dwarf galaxies and stellar streams in the halo of the Milky Way (for example, Li et al. 2019; Marshall et al. 2019), and rare transients such as merging neutron stars (Palmese et al. 2017), superluminous SNe (Smith et al. 2018; Angus et al. 2019), and rapidly evolving transients (Pursiainen et al. 2018).

Since 2012, OzDES has been targeting sources in the 10 DES deep fields with the $2 \mathrm{dF}$ fibre positioner on the AAT. As shown in fig. 1 of Yu15, the fields of view of $2 \mathrm{dF}$ and DECam are very similar. In addition to the 10 DES deep fields, OzDES targeted the DES MaxVis field during one of the OzDES observing runs. Centred at $\mathrm{RA}=97.5^{\circ}$ and $\mathrm{Dec}=-58.75^{\circ}$, it is observable during the entire DES observing season. It is one of the DES standard star fields that was observed during twilight. Yu et al. (2018) used quasars in this field to study continuum RM.

The scientific aims of OzDES focus on two areas: (i) using redshifts and distances of type Ia SNe to measure the expansion history of the Universe and thereby constrain the dark energy equationof-state parameter; and (ii) measuring the time lags between the continuum and the broad lines in a sample of AGN to measure their central black hole masses and to investigate the possibility of using AGN as a luminosity distance indicator that would explore a redshift range that is currently beyond the reach of SNe.

Over the 6 years that OzDES ran, OzDES obtained redshifts for 7000 candidate SN hosts (D'Andrea et al. 2018) and spectroscopically confirmed several hundred SNe. These redshifts will be used with distances to the SNe inferred from broad-band light curves obtained by DES to measure the expansion history of the Universe and to constrain the dark energy equation-of-state parameter. Using 
an initial sample of 207 spectroscopically confirmed type Ia SNe from the first 3 years of data in combination with 122 low-redshift $\mathrm{SNe}$ from the literature and constraints from the cosmic microwave background, DES (2019b) obtained a 7 percent constraint on a constant dark energy equation-of-state parameter. Analysis of the full DES sample is ongoing, and the final cosmological results will include approximately an order of magnitude more $\mathrm{SNe}$, the majority of which will be classified photometrically.

At the same time, OzDES has monitored a sample of 771 AGN up to $z \sim 4$ with the goal of measuring black hole masses using the time lag between variations in the continuum, measured from the broad-band photometry obtained by DES, and the broad lines, measured from the spectra obtained by OzDES. The first time lags, based on the first 4 years of data, were published in Hoormann et al. (2019).

In addition to these two main projects, OzDES is facilitating the studies of a number of areas, both within DES and outside of DES, by obtaining redshifts of targets of interest in these 10 fields using the 'spare' fibres that are not allocated to an AGN, an active transient, or a host galaxy. The most notable examples are: radio galaxies from the ATLAS survey (Franzen et al. 2015); brightest cluster galaxies (Webb et al. 2015); redMaGiC galaxies (Rozo et al. 2016; Prat et al. 2018); and luminous red galaxies (LRGs), which are used in combination with redshifts from other sources to train photometric redshift algorithms (Sánchez et al. 2014; Bonnett et al. 2016; Gschwend et al. 2018).

\section{OZDES OPERATIONS FOR Y4, Y 5 AND Y6}

Starting in 2013, OzDES ran for six observing seasons, with an initial allocation of 12 nights in Y1 (AAT semester 2013B), and allocations of 16,20,20,20, and 12 nights in subsequent years. In 2012, when aspects of the DES survey strategy were being tested during a period of science verification (SV), five nights were allocated to an OzDES precursor survey. As for DES, these five nights were used to develop and test the observing strategy that would be used for the next 6 years.

Observations typically started in late August or early September and terminated four to five months later in December or January the following year. The observing log for Y4, Y5, and Y6 can be found in Appendix $\mathrm{A}^{3}$ and follows the format presented for earlier years in Yu15 and Ch17.

In total, there were 41 numbered runs, with the last night of run 41 occurring on 2019 January 9. Some of the nights during these runs were shared with other programmes, such as 2dFLens (Johnson et al. 2017) during Y2 and Y3, XXL (Pierre et al. 2016) in Y4, DEVILS (Davies et al. 2018) in Y5, and the HSC Transient Survey (Yasuda et al. 2019) in Y6. During Y2 and Y3, OzDES and $2 \mathrm{dFLens}$ shared a common run numbering scheme, and there are a number of runs during those years in which only 2 dFLens fields were observed.

The end of Y5 marked the end of the DES programme to discover transients in the 10 DES deep fields. DES continued to target fields in the wide survey during Y6. OzDES also continued into a sixth year, but was no longer targeting transients. Instead, OzDES continued with its programme to monitor AGN (King et al. 2015; Hoormann et al. 2019) and to obtain redshifts of galaxies that hosted transients in earlier years. DES continued to target the 10 DES SN fields during Y6, but with a reduced frequency of about once a month.

\footnotetext{
${ }^{3}$ Appendices are available online.
}

These data are used to spectrophotometrically calibrate the spectra of the AGN in the deep fields that were being monitored by OzDES.

\subsection{Instrumental set-up}

Apart from the final OzDES run in Y6 (run 041), the instrumental set-up used in Y4, Y5, and Y6 was identical to the set-up used in Y3. The set-up in Y1 and Y2 and the precursor survey were slightly different. During these years, the central wavelength of the blue grating was set $20 \AA$ to the blue. Details of the set-up and the reasons for the change between $\mathrm{Y} 2$ and $\mathrm{Y} 3$ can be found in Ch17.

The final OzDES run was not officially scheduled as an OzDES run. At the beginning of 2019, an opportunity arose to take additional data in the $\mathrm{C} 3$ and $\mathrm{X} 3$ fields during time that was allocated to the HSC Transient Survey, which has similar aims to the SN programme in OzDES, but is targeting fainter, more distant host galaxies. For this run, and this run only, we used the set-up used by the HSC Transient Survey, which uses the x6700 dichroic (OzDES uses the $\mathrm{x} 5700$ dichroic) and a wavelength setting that results in a wavelength coverage that is shifted about $1000 \AA$ to the red, from 3700-8800 to $4700-9800 \AA$.

\subsection{OzDES target allocation}

OzDES targeted a wide range of sources over the 6 years it ran, with active transients, AGN, and host galaxies with $m_{\mathrm{r}}<24$ having the highest priority and occupying most of the fibres. A full listing of the source types that were observed in the last 3 years, together with their priorities, is provided in Table 1.

In addition to a priority, each type had a quota. Starting with the highest priority (priority 8), targets were randomly selected until there were no more targets to select or the quota for that type of source had been reached. Further details on the target selection algorithm are described in Y15.

If conditions were poor because of low transparency (generally more than 2 mag of extinction) and/or poor seeing (generally poorer than 3 arcsec), we switched to a backup programme that mostly consisted of bright galaxies. However, transients and AGN in the RM programme were always targeted.

Over the seasons, the composition of sources and their priorities changed. The biggest changes occurred at the end of Y5 and the beginning of Y6. In Y6, no new transients were being discovered, as DES stopped monitoring the 10 deep fields for transients; however, during Y6, we did continue to observe a small number of transients that were discovered in earlier years.

There were other changes too. At the end of Y5, the quota for SN hosts increased from 100 (200 for X3 and C3, the two extra deep fields) in the first 5 years to 200 (250 for X3 and C3), and two new classes of sources were added. Given the success at obtaining redshifts for sources as faint as $r_{\mathrm{AB}} \sim 24$ and no strong evidence to a floor in the noise (Ch17), we added galaxies with $24<r_{\mathrm{AB}}<25$. We labelled these as SN_host_faint. We had targeted hosts as faint as this in past seasons, but the aim then was different. Then, if we did not see an emission line that enabled us to get a redshift after one run, we discarded the source. During Y6, we continued observing these faint hosts until a secure redshift (see Section 4) was obtained.

We also added host galaxies of transients that were previously observed when the transient was still bright enough to contribute to the flux density. We labelled these as SN_free_host. We will use these spectra to examine the properties of host galaxies without light from the $\mathrm{SN}$, and to subtract the galaxy spectrum from earlier spectra 
Table 1. A listing of the source types observed during Y4, Y5, and Y6, together with the nominal priority. The list is ordered in priority from highest (8) to lowest (1). Also listed are source types (noted with asterisks) from the first 3 years of OzDES that were discontinued before the start of Y3. Source types for the MaxVis field are listed in Appendix C.

\begin{tabular}{|c|c|c|}
\hline Type & Priority & Comment \\
\hline Transient & 8 & Active transients \\
\hline AGN & 7 & Reverberation mapping programme (Hoormann et al. 2019) \\
\hline SN_host ${ }^{a}$ & 6 & Hosts with $m_{\mathrm{r}}<24$ \\
\hline Cooke_host & 6 & Host galaxy candidates of transients found in deep $\mathrm{SNLS}^{b}$ stacks \\
\hline Strong_Lens* & 6 & Candidate strong lenses (Nord et al. 2016; Jacobs et al. 2019) \\
\hline White Dwarfs* & 6 & White dwarfs to aid calibration \\
\hline DEVILS $^{c}$ & 5 & Objects in the DEVILS survey (Davies et al. 2018) \\
\hline SNLS & 5 & SN hosts from the SNLS survey data (Astier et al. 2006; Betoule et al. 2014) \\
\hline Tertiary* & 5 & Stars with a broad range of colours to aid calibration \\
\hline Cluster Galaxies & 4 & Cluster Galaxies (some targets were observed at higher priority) \\
\hline Radio Galaxies & 4 & Radio Galaxies (some targets were observed at higher priority) \\
\hline QSO & 4 & Faint QSOs in the S1 and S2 fields \\
\hline XXL_QSO & 4 & QSOs in the XXL fields (Pierre et al. 2016) \\
\hline SN_host_faint & 4 & Hosts with $24<m_{\mathrm{r}}<25$ \\
\hline RedMaGiC & 4 & For calibrating photometric redshifts of redMaGiC galaxies (Rozo et al. 2016) \\
\hline $\mathrm{SpARCS}^{d}$ & 4 & Brightest Cluster Galaxies from the SpARCS survey (Wilson et al. 2009) \\
\hline ELG* $^{*}$ & 4 & Emission Line Galaxies \\
\hline SN_free_host & 3 & Host galaxies of previously targeted transients \\
\hline Photo- $z$ & 3 & For training and testing photometric redshift algorithms \\
\hline LRG & 2 & Luminous Red Galaxies \\
\hline Bright galaxies & 1 & Backup targets during poor conditions \\
\hline Bright stars* & 1 & Backup targets during poor conditions \\
\hline
\end{tabular}

Notes. ${ }^{a}$ The type 'SN host' not only includes galaxies that hosted an $\mathrm{SN}$, but also galaxies that hosted transients that were not SNe.

${ }^{b}$ SNLS - Supernova Legacy Survey.

${ }^{c}$ DEVILS - Deep Extragalactic Visible Legacy Survey.

${ }^{d}$ SpARCS - Spitzer Adaptation of the Red-Sequence Cluster Survey.

Table 2. Fibre allocations for the last 3 years of OzDES (Y4, Y5, and Y6).

\begin{tabular}{|c|c|c|c|c|c|c|c|}
\hline \multirow[b]{2}{*}{ Object } & \multicolumn{2}{|c|}{ Y4 } & \multicolumn{2}{|r|}{ Y5 } & \multicolumn{3}{|c|}{ Y6 } \\
\hline & $\begin{array}{l}\text { Fibre } \\
\text { hours }\end{array}$ & $\begin{array}{l}\text { Fraction of } \\
\text { fibre hours }\end{array}$ & $\begin{array}{l}\text { Fibre } \\
\text { hours }\end{array}$ & $\begin{array}{l}\text { Fraction of } \\
\text { fibre hours }\end{array}$ & $\begin{array}{l}\text { Fibre } \\
\text { hours }\end{array}$ & $\begin{array}{l}\text { Fraction of } \\
\text { fibre hours }\end{array}$ & \\
\hline Transients & 939 & 2.7 per cent & 1195 & 3.2 per cent & 29 & 0.1 per cent & \\
\hline AGN & 8191 & 23.2 per cent & 8578 & 23.1 per cent & 6115 & 22.8 per cent & \\
\hline SN hosts & 13333 & 37.8 per cent & 14413 & 38.9 per cent & 18513 & 69.1 per cent & \\
\hline Cluster Galaxies & 1219 & 3.5 per cent & 1048 & 2.8 per cent & 449 & 1.7 per cent & \\
\hline Radio Galaxies & 1989 & 5.6 per cent & 1455 & 3.9 per cent & 0 & 0.0 per cent & \\
\hline DEVILS & 0 & 0.0 per cent & 720 & 1.9 per cent & 0 & 0.0 per cent & \\
\hline RedMaGiC & 2220 & 6.3 per cent & 3481 & 9.4 per cent & 0 & 0.0 per cent & \\
\hline LRGs & 4235 & 12.0 per cent & 3137 & 8.5 per cent & 0 & 0.0 per cent & \\
\hline Other & 3107 & 8.8 per cent & 3071 & 8.3 per cent & 1688 & 6.3 per cent & \\
\hline Total & 35232 & - & 37097 & - & 26793 & & - \\
\hline
\end{tabular}

that contained light from the both the galaxy and the transient. The aim here is to see if one can identify the type of transient in spectra where this was not possible. This has been proven to work for spectra taken with slits (Dawson et al. 2009), but it has never been tried for spectra obtained with fibres.

Fibre allocations for the last 3 years of OzDES are listed in Table 2 and shown as plots in Fig. 1. A similar table for the first 3 years is provided in Ch17. After Y1, a quarter of all fibres were allocated to AGN that were being followed in the OzDES RM. The number of fibres allocated to SN hosts steadily increased from 12 percent in Y1 to 39 percent in Y5. The large jump from Y5 to $\mathrm{Y} 6$ in the number of SN hosts reflects the increased quota for
SN hosts in $\mathrm{Y} 6$ and the addition of two new SN host categories. Almost all fibres in $\mathrm{Y} 6$ were allocated to an AGN or an SN host.

Fibre allocations over the full OzDES survey are shown in Fig. 2. The three most commonly observed sources were SN hosts ( 35 per cent), AGN (26 per cent), and galaxies used to train photometric redshift algorithms: LRGs, ELGs, and redMaGiC galaxies (21 per cent).

There can be considerable overlap in the individual target catalogues that make up the OzDES target input catalogue. For example, a SN host can also be listed as a radio galaxy, LRG, or redMaGiC galaxy. In Table 2 and Figs 1 and 2, objects retain the 

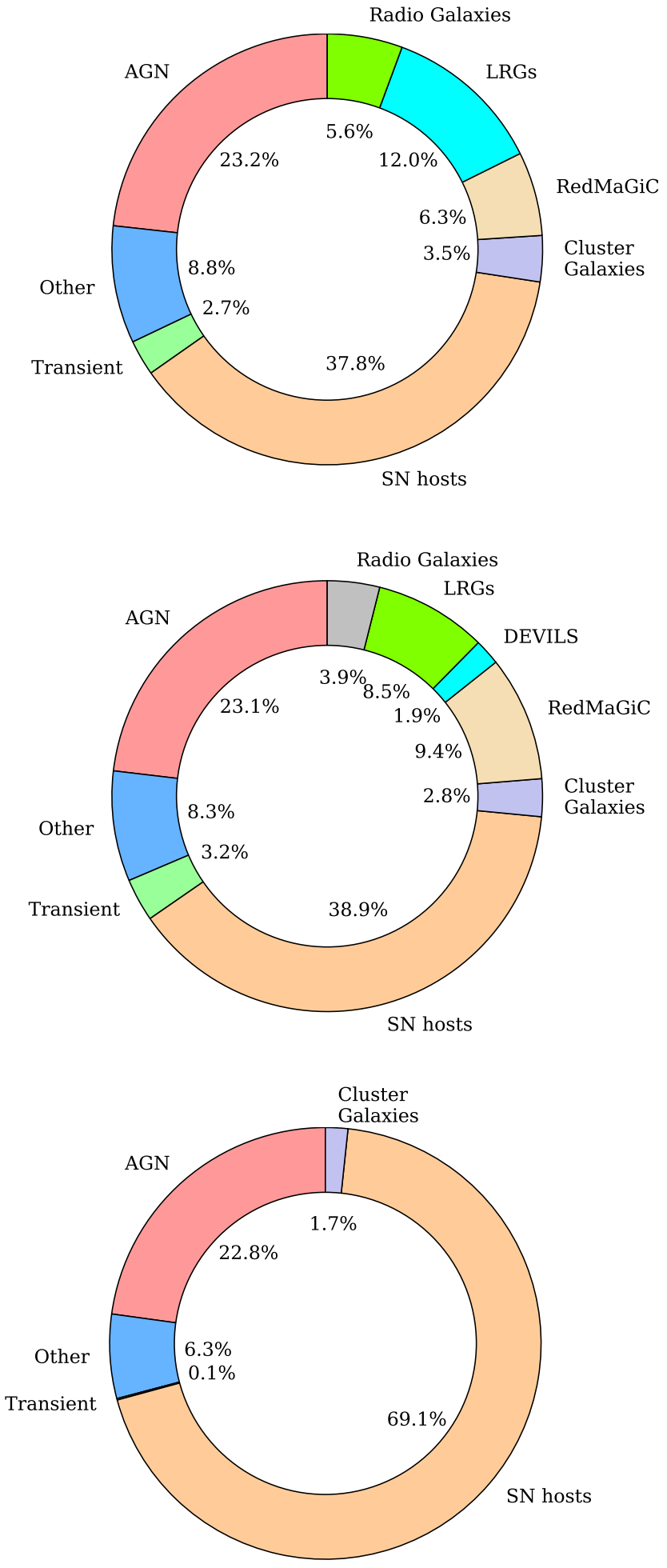

Figure 1. Plots showing how the allocation changed in the last 3 years of OzDES: Y4 at the top, Y5 in the middle, and Y6 at the bottom. The percentages represent the fraction of fibre hours. In the final year, almost all targets were either AGN or SN hosts.

type that has the highest priority. For example, a source that is listed in the OzDES target input catalogue as a SN host, radio galaxy, an LRG, and a redMaGiC galaxy will be classified and counted as a SN host.

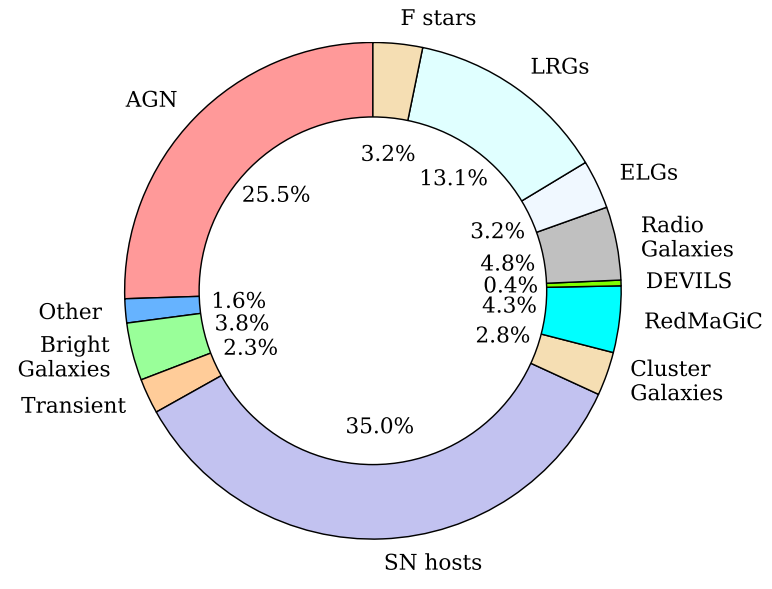

Figure 2. Plot showing the relative allocation for the full 6 years of OzDES. The percentages represent the fraction of fibre hours.

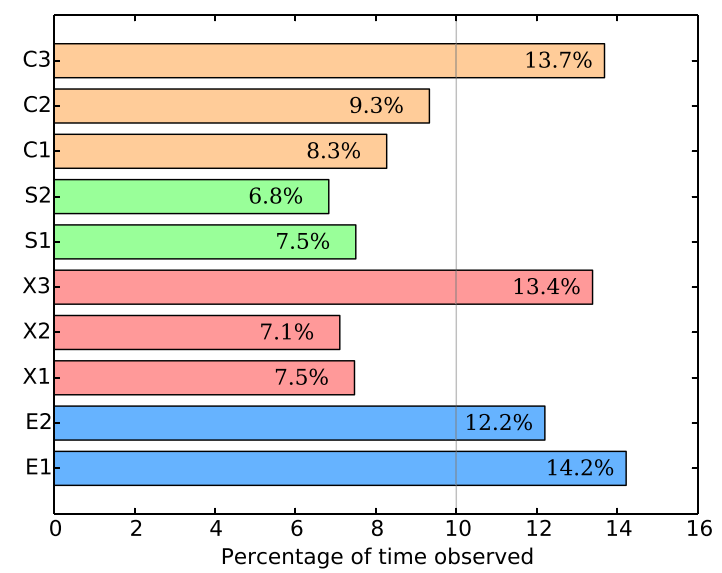

Figure 3. Bar plot showing the percentage of time each field was observed. The vertical line sits at 10 percent, which is where all fields would land if they had been observed with the same frequency. The X3, C3, E1, and E2 fields were the ones most frequently observed, reflecting the priorities these fields had and their locations in the sky.

\subsection{Observing strategy, calibration, and data reduction}

During each run, we aimed to get at least two 2400-s exposures per field. This was not always possible due to the length of some runs and weather conditions. Of the 31 runs that were scheduled for OzDES, we were able to observe all 10 fields on 16 occasions. There were only two, relatively short runs, where we did not observe any fields because of poor weather.

Overall, $\sim 25$ percent of time was lost to poor observing conditions. Another $\sim 5$ percent of the time was sub-optimal (thick cirrus and/or poor seeing), and was used for the backup programme. Observing logs for the final 3 years of OzDES, including the amount of time lost for poor weather, are included in the appendixes.

The two extra deep fields, C3 and X3, had the highest priority, so these fields were the most frequently observed, as can be seen in Fig. 3. The two E fields, E1 and E2, were also observed frequently, since these fields were often the only ones visible at the beginning of the night during the start of the observing season and are the most southern of the 10 deep fields, which results in a longer window of observability from the AAT. Next in priority were the six remaining fields: X1, X2, C1, C2, S1, and S2. 


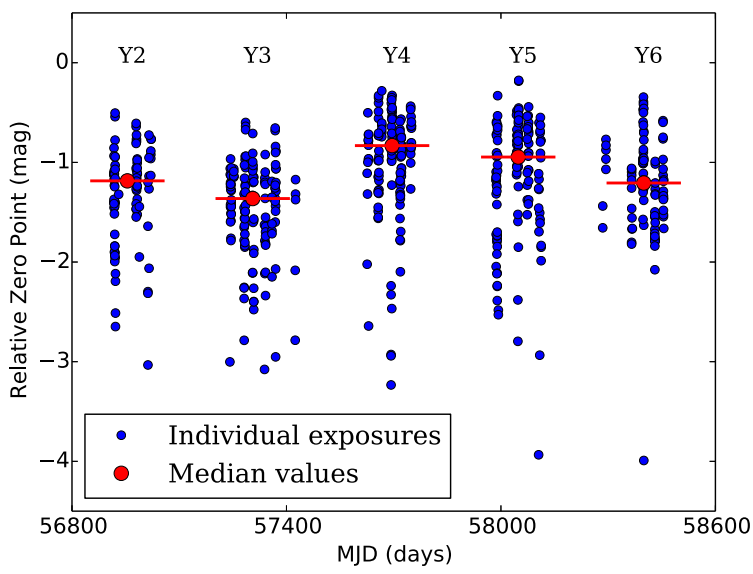

Figure 4. Plot of the relative throughput (zero-point) of data taken from Y2 to Y6. The median value for each year is shown as the red dot and the red line. The scale is in magnitudes and more positive numbers indicate higher throughput. In Y1, very few F stars were observed. Consequently, there are no reliable throughput measurements for the first year of OzDES. The relative zero-point plotted here differs from the zero-point recorded in the FITS headers by an additive constant of 33. See footnote d to Table B3 in the online version for details on how the zero-point is computed.

In addition to the sources listed in Table 1, about a dozen fibres per field were allocated to F stars, and another 25 'sky' fibres were placed on positions that were free of sources. The targeting priority for both the F stars and the 'sky' fibres was set to 5, which is below that of transients, AGN, and SN hosts, but higher than other types of objects. See Table 1 for details.

The F stars were primarily used to estimate throughput. The throughput was measured by integrating the extracted and wavelength calibrated spectra though the DECam filter band passes (Flaugher et al. 2015). For the red arm, we used the DECam $r$ filter: For the blue arm, we used the DECam $g$ filter. The relative throughput of the red arm over the course of OzDES is shown in Fig. 4. The throughput is reported in magnitudes, so each tick on the vertical access corresponds to a difference of about 2.5 in throughput. The vertical scatter is largely driven by transparency and atmospheric seeing.

Spectra that were taken when the throughput was excessively low (a couple of magnitudes lower than the medians shown in Fig. 4) are not used in the coadded spectra; however, they are included in the data release. For such spectra, the QC keyword in the FITS header is given the value poorConditions.

In the early years, we used the F stars to derive the transfer functions for the red and blue arms that were then used throughout the survey, and to develop techniques that used the broad-band DES photometry to spectrophotometrically calibrate the spectra of AGN used for the OzDES RM project (Hoormann et al. 2019).

All data were processed with the OzDES pipeline, which uses a modified copy of v6.46 of 2DFDR ${ }^{4}$ (Croom, Saunders \& Heald 2004) and our own bespoke PYTHON scripts. The steps are described in Yu15 and Ch17, so we do not repeat them here. Since Ch17, a small number of improvements to the pipeline have been made, the most significant of which were a more robust algorithm to determine the factor that scales spectra in the blue and red arms, modifications to the PCA sky subtraction algorithm to make it less sensitive to cosmic rays that were not fully masked, and more complete masking of artefacts. These artefacts are described in section B3.

All data were processed at the telescope, and combined with data taken during earlier observing runs. Once processed, all raw and reduced files were then archived on a server in Canberra. Typically, this was done daily, after the night had ended, but before the start of the next night. After each field was processed, the data were made available for OzDES team members ${ }^{5}$ dedicated to the task of measuring redshifts within $24-48 \mathrm{~h}$ of the data being taken. With the exception of transients and AGN, sources with secure redshifts (see the next section) were removed from the target catalogues and not re-observed during subsequent nights. During some of the longer OzDES runs, we observed a number of the DES fields on multiple occasions. If this occurred, then all objects that were observed on the first occasion would be given higher priority in subsequent observations, unless they had a secure redshift and had been removed from the target catalogue. We have reprocessed all the data with the same version of the pipeline on a couple of occasions. This ensures that all data are processed in a consistent manner. The latest version of the OzDES pipeline is version 18.21, and all data presented in this paper have been processed with that version.

\section{REDSHIFTS}

As noted in Ch17, redshifts have been measured using MARZ (Hinton et al. 2016) since the start of Y3. Earlier than this RUNZ, which was developed by Will Sutherland, had been used. Measuring redshifts is a task that is performed by two redshifters, whose results are collated, scrutinized and then merged by the chief redshift whip. Merging is also done using MARZ. The merged results then enter the OzDES data base, from which all other products are derived.

OzDES uses a redshift flag to quantify the confidence that the reported redshift is correct. As reported in Yu15 and Ch17, the flag can take on the following five main values:

(i) $Q=4$, redshift based on multiple strong spectroscopic features matched, $>99$ per cent confidence.

(ii) $Q=3$, redshift based typically on a single strong spectroscopic feature or multiple weak features, $>95$ per cent confidence.

(iii) $Q=2$, potential redshift associated with typically a single weak feature, low confidence, not to be used for science.

(iv) $Q=1$, no matching features, thus no constraints on redshift.

(v) $Q=6$, securely classified star.

As the survey progressed, we monitored the growing redshift catalogue for sources that had conflicting redshifts. These are sources in which the redshift changes between observing runs but the redshift quality flag, $Q$, remains unchanged and $Q>2$. An example is a source that is assigned a $Q=3$ redshift during one run, but with the addition of more data in a later run, it is assigned a different redshift $(\Delta z>0.05)$ with the same quality flag.

On two occasions, once at the end of Y4 and once at the end of Y6, we collated all cases where there was a conflict, defined on these occasions having a redshift difference larger than 0.05 , and reprocessed and re-redshifted these sources in the same way we redshifted sources during each observing run. The revised redshifts were then added to our redshift catalogue. At the end of Y4, there were a total of 191 sources that had conflicting redshifts. At the end $\mathrm{Y} 6$, there were a total of 49 , perhaps illustrating a degree of improvement by the human redshifters. ${ }^{4}$ More information on 2DFDR is available from https://aat.anu.edu.au/scien
ce/software/2dfdr.
${ }^{5}$ Colloquially referred to as 'redshifters'. 


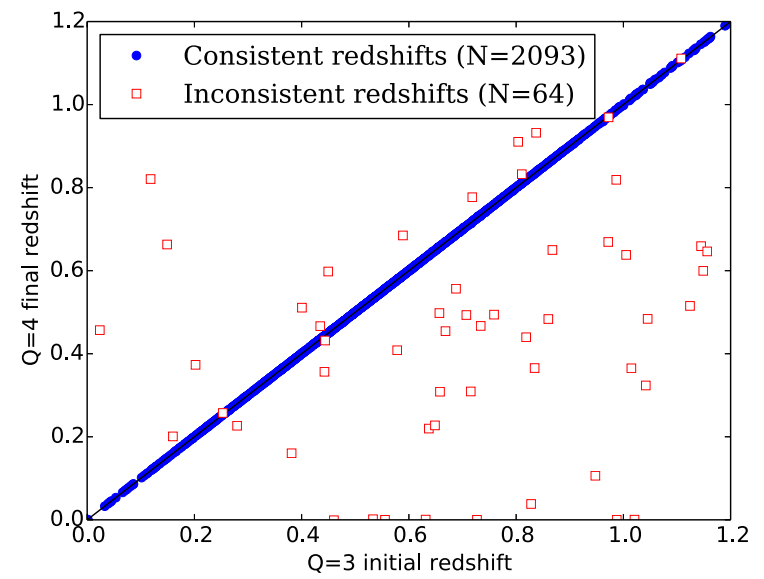

Figure 5. Redshift comparison for OzDES SN hosts that attained a $Q=$ 3 quality redshift before attaining a $Q=4$ quality redshift after further observations. Outliers (defined as $\Delta z>0.003$ ) are shown as red squares. Sources with concordant redshifts are shown as blue points. The black line represents the one-to-one relationship. Only 3.1 per cent of sources changed redshifts when moving from $Q=3$ to 4 .

In preparation for the second OzDES data release, we revisited cases with conflicting redshifts, this time decreasing the threshold from 0.05 to 0.01 in redshift. We also included sources that were classified with $Q=3$ quality redshift at some stage during the survey but ended up with a lower redshift quality flag once all data were combined. Combined, about 820 sources were re-examined.

\subsection{Redshift reliability}

Using data taken up until the end of Y1, Yu15 estimated the reliability of redshifts that are assigned $Q=3$ and 4 using external catalogues and repeat observations of the same target. They find that $Q=4$ quality redshifts are 100 per cent reliable and $Q=3$ quality redshifts are $\sim 90$ per cent reliable. After Y1, a number of measures were put in place to lift the reliability of the $Q=3$ quality redshifts. Our target for $Q=3$ quality redshifts at the start of the OzDES survey was $\sim 95$ per cent. As discussed below, we have reached this goal.

Ch17 used a different approach to assess redshift reliability, taking advantage of the observing strategy applied to SN hosts. Unlike other target types, SN hosts were not removed from the input target catalogues until they attained a $Q=4$ quality redshift. For those $\mathrm{SN}$ hosts with $Q=4$ quality redshifts that initially attained a $Q=3$ quality redshift, one can compare the initial redshift with the final redshift and get an estimate of reliability of the $Q=3$ quality redshifts, assuming that the $Q=4$ quality redshifts have a known reliability, which we take to be 100 percent, following that found in Yu15.

Using data up until the end of $\mathrm{Y} 3, \mathrm{Ch} 17$ found that the redshifts of 17 out of 443 sources ( 3.8 per cent) changed between the initial $Q=$ 3 quality redshift and the final $Q=4$ quality redshift. We repeated this analysis, using a sample that is almost five times larger. We find that 64 of 2093 sources (3.1 per cent) changed redshifts, a slightly smaller fraction than that found in Ch17. The results are shown in Fig. 5.

The $\mathrm{SN}$ hosts allow one to examine the reliability of the $Q=$ 3 quality redshifts in a second, complementary way. A number of the fainter $\mathrm{SN}$ hosts have been observed over several years and 20 or more observing runs. As more data were obtained and added to earlier data, they would have been re-redshifted, sometimes twice or more per run, which can occur if they were observed over multiple times during a run. Some sources have dozens of redshift measurements. Every time a source was redshifted, there is a chance of an erroneous redshift being assigned to that source. We can get a rough estimate of how often this happens by searching for objects that obtained a $Q=3$ quality redshift during one run, but did not subsequently obtain a $Q=3$ or 4 quality redshift with more data.

We search the data base for examples where a $Q=3$ quality redshift was assigned once and not assigned again, even if the exposure time had increased by a factor of 2 and then by a factor of 3 by the end of Y6. For these two cases, we identified 31 and $11 \mathrm{SN}$ hosts, respectively. For these sources, we reduce the redshift quality flag from $Q=3$ to 2 . However, this can only be applied to $\mathrm{SN}$ hosts, as all other objects are deselected from the input target catalogue once a $Q=3$ quality redshift is obtained. There are 1100 SN hosts with a $Q=3$ quality redshift, potentially indicating an error rate in addition to that noted above of 1-3 per cent.

The SN hosts also allow one to get a handle on the repeatability of the redshift measurement. We first selected SN hosts that have at least four redshift measurements with a quality flag of 3 or 4 . We then compute the RMS of the redshifts for each host. The median RMS is 0.00015 . While this is indicative of the redshift uncertainty, it is likely that it is a lower limit, as the measurements are not fully independent: redshifts derived from data taken later include earlier data. Indeed, the median RMS is a factor of 2 smaller than the uncertainty reported in Yu15, who report 0.0003 for SN hosts using redshifts that were obtained from data that were fully independent.

The impact of a redshift uncertainty of 0.0003 on the dark energy equation-of-state parameter is negligible if the uncertainty is not systematic. If the error is systematic, then the dark energy equationof-state parameter could be biased (Calcino \& Davis 2017).

\subsection{Redshift outcomes}

The diversity of objects that were observed over the 6 years by OzDES is large, from radio sources covering a broad range in magnitude and redshift, to more restricted classes of objects, such as LRGs, covering smaller magnitude and redshift ranges.

In Figs 6-8, we provide magnitude and redshift distributions for RedMaGiC galaxies, LRGs, and radio galaxies. As noted at the end of Section 3.2, some targets had multiple types. An LRG may have been classified as a redMaGiC galaxy and vice versa. About 40 percent of all sources observed by OzDES had multiple types. In these plots, we include all objects of the indicated type, even if it also had another type.

Also shown as an insert to these figures and Fig. 10 is the redshift completeness, defined as the number of objects with a redshift divided by the number of objects that were targeted. A number of targets were spectroscopically classified as stars. These were removed before computing the redshift completeness.

\subsection{Redshift completeness}

\subsubsection{Completeness as a function of magnitude and exposure time}

A little more than one third of the 192000 fibre hours that were spent observing all targets were spent on SN hosts (see Table 3). SN hosts were targeted during the entire duration of OzDES and were not deselected from the input target catalogue until a $Q=4$ quality redshift was obtained. They are therefore useful in examining how redshift completeness depends on magnitude and exposure time. 

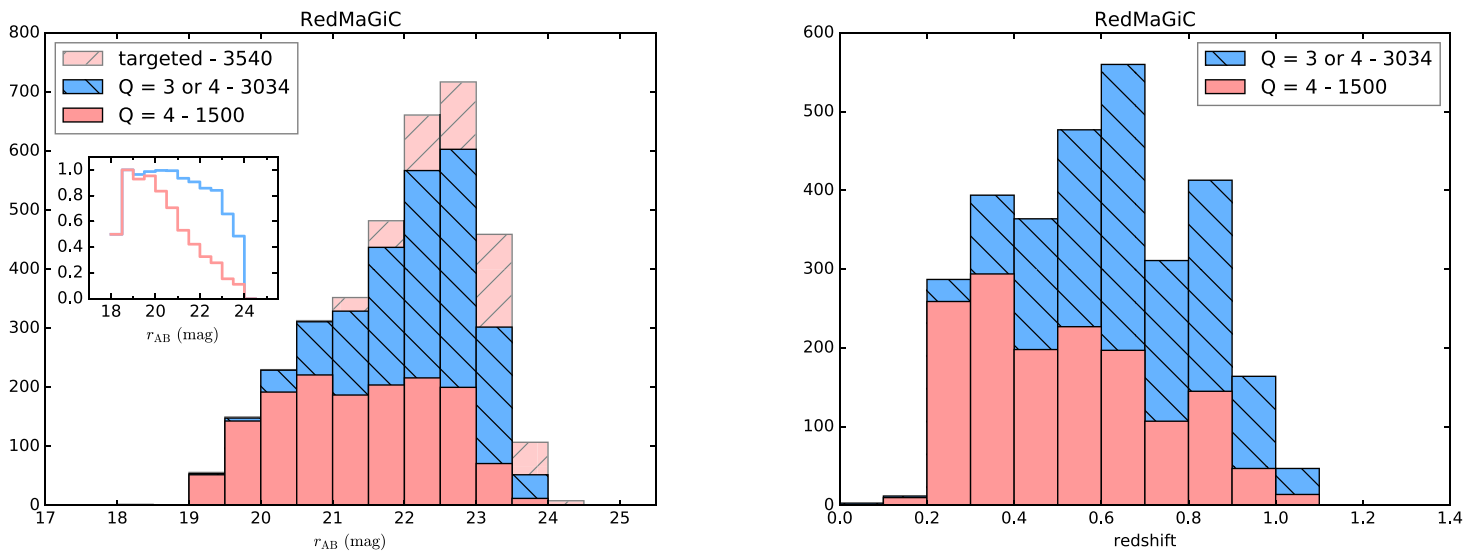

Figure 6. Left: The number of redshifts as a function of $r_{\mathrm{AB}}$ for redMaGiC galaxies. The inset shows the fractional completeness as a function of magnitude. The redshift completeness is the number of objects with $Q=3$ (or 4) divided by the number of objects that were targeted. For all object types other than host galaxies, sources were removed from the target input catalogues once they reached a $Q=3$ redshift quality. Right: Redshift histogram. RedMaGiC galaxies tend to be massive galaxies with little ongoing star formation, so their spectra are usually devoid of strong emission lines. Most of the redshifts were obtained from the calcium $\mathrm{H}$ and $\mathrm{K}$ lines in absorption, which are usually quite distinct in redMaGiC galaxies.
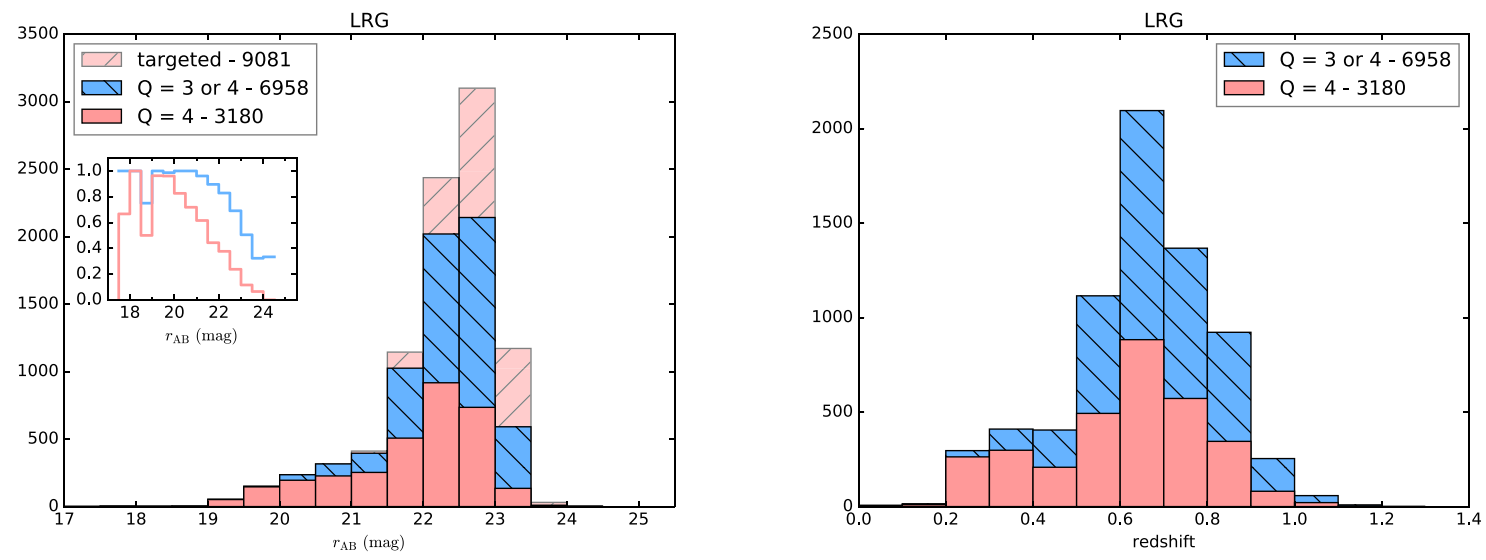

Figure 7. As for Fig. 6 but for Luminous Red Galaxies. LRGs, like redMaGiC galaxies, also tend to have little ongoing star formation, so the spectra are usually devoid of strong emission lines.
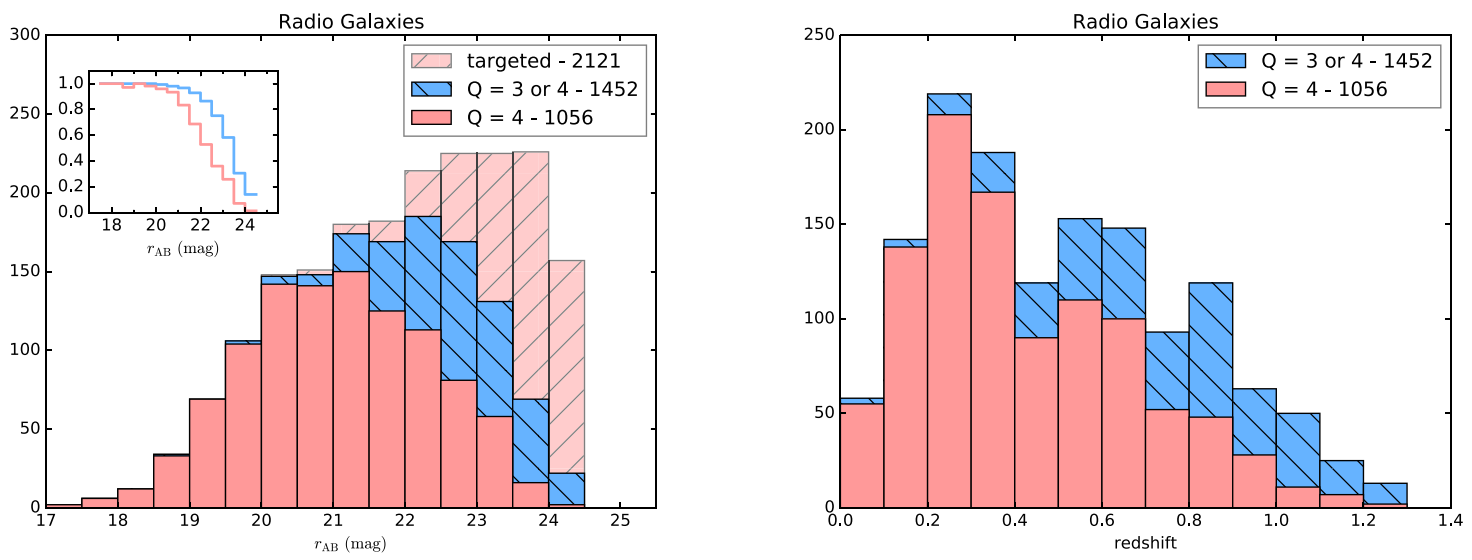

Figure 8. As for Fig. 6 but for radio galaxies.

However, caution is needed before applying the results from $\mathrm{SN}$ hosts to the general population of field galaxies, because SN hosts are a biased subset. SN hosts are more likely to be $z<1.35$, and therefore within the spectral range covered by OzDES.
All SN hosts were selected on the basis of the detection of a transient in the DECam images. With the exception of superluminous $\mathrm{SNe}$, which are rare, there would be few SN hosts that are at redshifts that place the [O II] $\lambda 3727 \AA$ doublet beyond the 


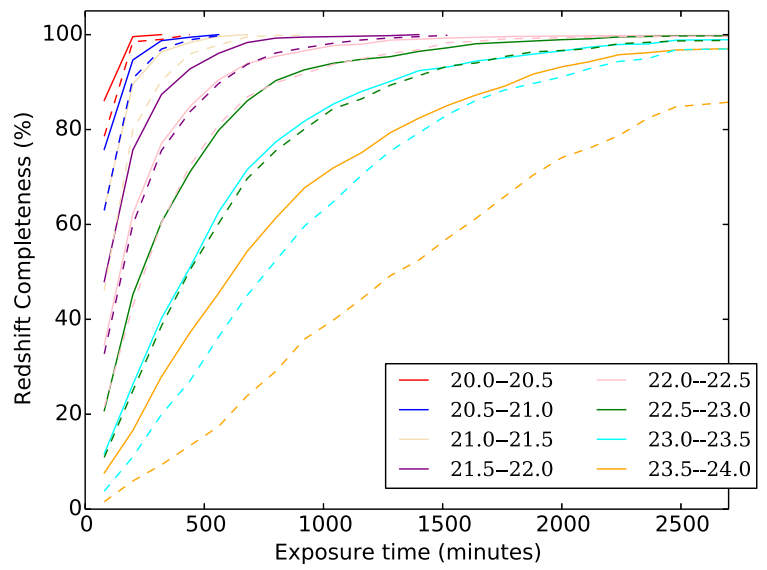

Figure 9. Redshift completeness as a function of exposure time for different magnitude bins and two redshift quality flags. The dashed lines represent redshift quality 4 and the solid lines represent redshift quality 3 . The lines end once completeness reaches 100 per cent.

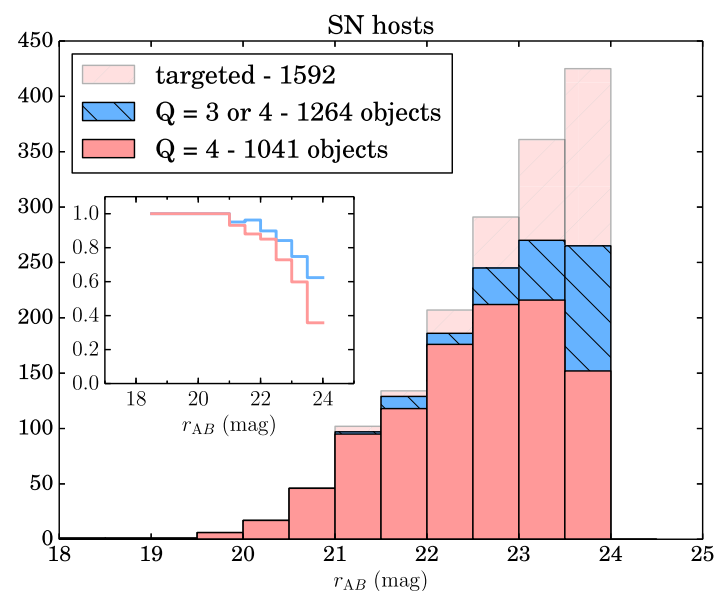

Figure 10. The number of SN hosts with redshifts binned by $r_{\mathrm{AB}}$. The redshift completeness is shown in the insert. Faint SN hosts (i.e. sources classified as SN_host_faint) are not included. Throughout the survey, over 7000 objects were targeted as potential SN hosts. Plotted here are the ones most likely to be SNe Ia. Excluded from this plot are 279 SN hosts that have redshifts from external surveys, as these objects were not targeted. The last bin would reach 95 per cent completeness if we were able to integrate for $40 \mathrm{~h}$ on all sources, as shown in Fig. 9.

red end of the spectral range covered by the setting we used in AAOmega, which is at $8800 \AA$. The red end corresponds to a redshift of $z \sim 1.35$ for [O II]. Nearly all of the SN Ia detected by DES will be below this redshift (Bernstein et al. 2012). Hence, when interpreting the completeness of OzDES as a function of magnitude, the completeness will be different than that for a survey that targeted field galaxies.

The redshift completeness of SN hosts as a function of exposure time is shown in Fig. 9. The completeness is shown for different magnitude bins and two redshift quality flags. As expected, completeness increases with exposure time and is highest for the brightest sources. It is also higher for lower quality redshift flags.

For the faintest bin $\left(23.5<r_{\mathrm{AB}}<24\right)$, the redshift completeness reaches $\sim 95$ per cent for quality 3 redshifts after $40 \mathrm{~h}$ of integration. This does not mean that we reached this level of completeness in
Table 3. Fibre allocations for the entire OzDES survey.

\begin{tabular}{lcr}
\hline & \multicolumn{2}{c}{ All years } \\
Object & $\begin{array}{c}\text { Fibre } \\
\text { hours }\end{array}$ & $\begin{array}{c}\text { Fraction of } \\
\text { fibre hours }\end{array}$ \\
\hline Transients & 4438 & 2.3 per cent \\
AGN & 49548 & 25.5 per cent \\
SN hosts & 68028 & 35.0 per cent \\
Cluster Galaxies & 5523 & 2.8 per cent \\
DEVILS & 720 & 0.4 per cent \\
Radio Galaxies & 9271 & 4.8 per cent \\
RedMaGiC & 8394 & 4.3 per cent \\
F stars & 6247 & 3.2 per cent \\
LRGs & 25521 & 13.1 per cent \\
ELGs & 6175 & 3.2 per cent \\
Other & 3043 & 1.6 per cent \\
Bright Galaxies & 7298 & 3.8 per cent \\
Total & 194207 &
\end{tabular}

the survey, as not all SN hosts have been observed for that long. We anticipate that if we were to observe this long for all sources, then we would reach this level of completeness. As shown in Fig. 10, the completeness in the faintest bin is 65 percent.

\subsubsection{Completeness as a function of plate position}

The positioning of fibres relies on a series of transformations between sky coordinates and plate coordinates. The transformations include the poscheck, which is updated by observatory staff, typically during the first night after $2 \mathrm{dF}$ is remounted on the telescope, and the plate survey, which is run every time a field is configured. The accuracy of these transformations has a direct impact on the amount of flux entering fibres and on the redshift completeness.

We examine how the redshift completeness varies across the field for SN hosts and LRGs. For LRGs, we include redMaGiC galaxies. Any variation in the redshift completeness across the field would be indicative of a problem in the accuracy of the transformations.

In the two panels of Fig. 11, we plot the position of SN hosts with and without redshifts, respectively. One can clearly see the boundary of the DECam chips traced by the SN hosts. The 10 fields in the DES deep survey were observed with minimal dithering. Consequently, chips gaps between the DECam CCDs were not covered and so no $\mathrm{SNe}$ are found in these gaps. A deficit of $\mathrm{SN}$ hosts can be seen in areas where CCDs were not functioning for some fraction of the survey.

Similarly, in the two panels of Fig. 12, we plot the position of LRGs with and without redshifts. The boundary of the DECam chips can no longer be seen, as LRGs were selected from imaging data that were more continuous.

The redshift completeness is shown for SN hosts and LRGs in Fig. 13. The completeness is not uniform for either class of object. In both cases, there is a drop towards the edge of the field. This is not unexpected and is due to a drop in the image quality delivered by the $2 \mathrm{dF}$ corrector at the edge of the field and to the accuracy at which the plate can be rotated, which affects fibre near the field edge more than fibres in the centre. Less expected is a gradient from lower left to upper right in the redshift completeness of LRGs.

In Fig. 14, we show the average number of exposures it takes to get a redshift. There is a general trend of increasing exposure time as one moves from the centre of the field of view to the edges. Again, the LRGs show a more significant gradient than the SN hosts. 

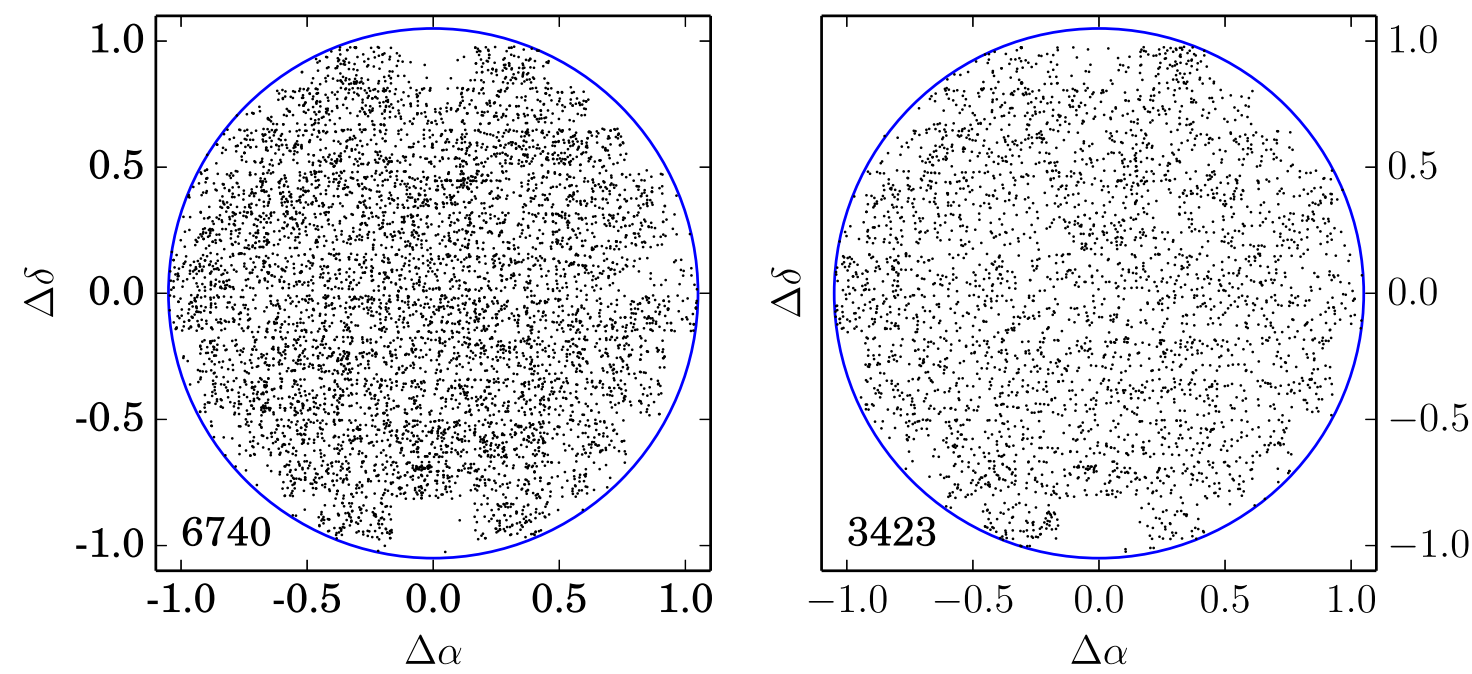

Figure 11. The position, with respect to the centre of the $2 \mathrm{dF}$ field plates, of $\mathrm{SN}$ hosts with redshifts (left plot) and without redshifts (right plot). The blue circle represents the $2.1 \mathrm{deg}$ diameter of the $2 \mathrm{dF}$ field of view. Notable in these images are the chip gaps, the boundary of the mosaic, and the two CCD chips at the bottom and top of the mosaic that were not operational for some part of DES. In this plot north is up and east is to the right. Object numbers are given in the lower left corners.
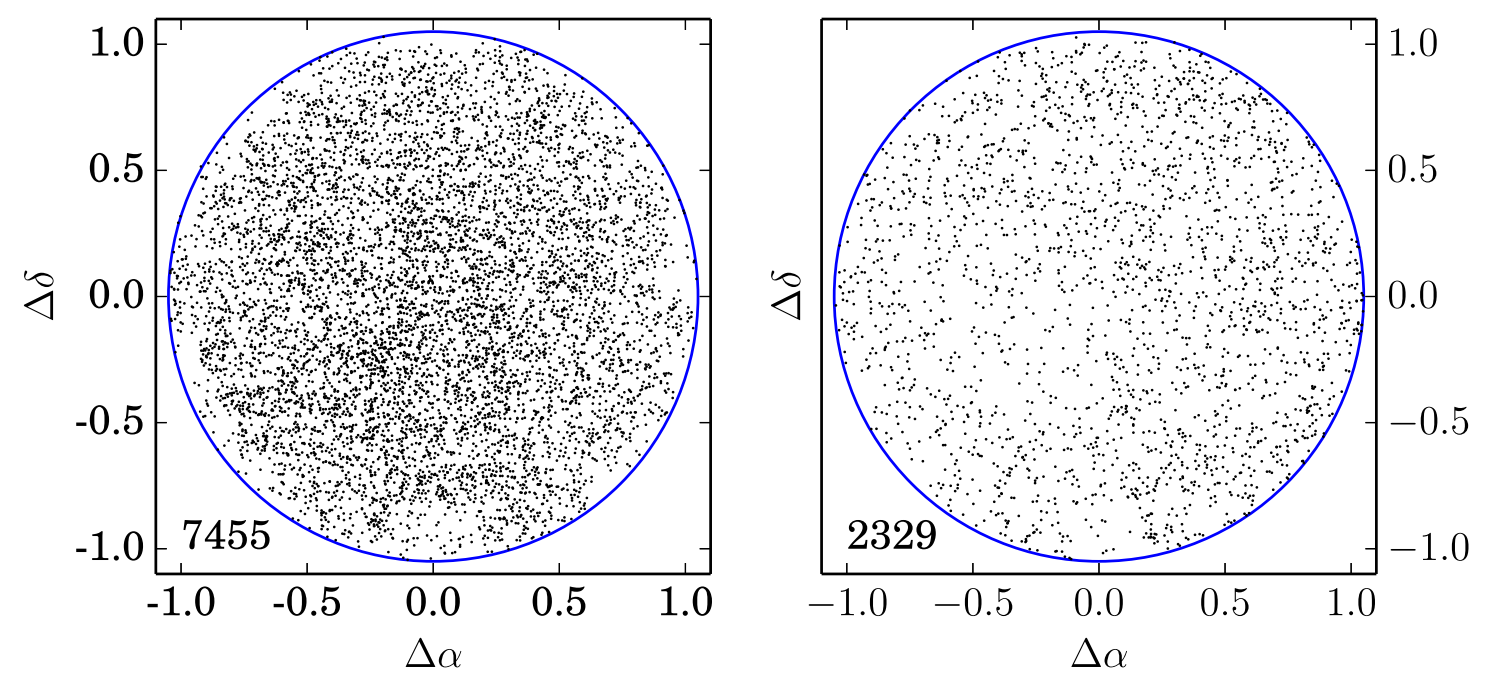

Figure 12. The position, with respect to the centre of the $2 \mathrm{dF}$ field plates, of LRGs with redshifts (left plot) and without redshifts (right plot). Gone are the chip gaps. The gap left by the chip at the bottom of the mosaic is still visible. Unlike the plot for SN hosts, there appears to be a gradient, which becomes clearer in Fig. 13. Object numbers are given in the lower left corners.

There are a couple of potential reasons why the gradient is less significant for SN hosts. First, SN hosts are not removed from the target catalogue until a secure, quality 4 redshift is obtained, whereas LRGs are removed from the target catalogue once a quality 3 redshift is obtained. This will reduce field-dependent redshift incompleteness. Secondly, the flux lost due to fibre positioning errors is more severe for point sources than extended objects. LRGs due to their higher redshift and redder colours will be more point like than SN hosts. At the AAT, the profile of sources at these redshifts is dominated by the seeing, which will be better in the red than the blue.

The gradient has no impact on the primary OzDES science goals, as the spatial location of the AGN and SNe are not relevant, but the gradient needs to be taken into account if these data were to be used to estimate clustering from statistics such as the two-point galaxy-galaxy correlation function.

\section{THE SECOND OZDES DATA RELEASE}

This paper marks the second OzDES release, which will be referred to as OzDES-DR2. OzDES-DR2 differs from OzDES-DR $1,{ }^{6}$ the first OzDES data release, in two key aspects.

(i) The redshift catalogue includes all objects observed during the 6 years of OzDES and the OzDES pre-survey. This includes SN hosts, $\mathrm{SNe}$, and targets for which a redshift could not be obtained.

(ii) The fully processed spectra are provided, including all individual spectra, as well as the stacked spectrum of each object.

\footnotetext{
${ }^{6}$ The redshift catalogue for OzDES-DR1 that is available from Data Central was described in Ch17 and contained redshifts of spectroscopically confirmed sources. No spectra were released.
} 

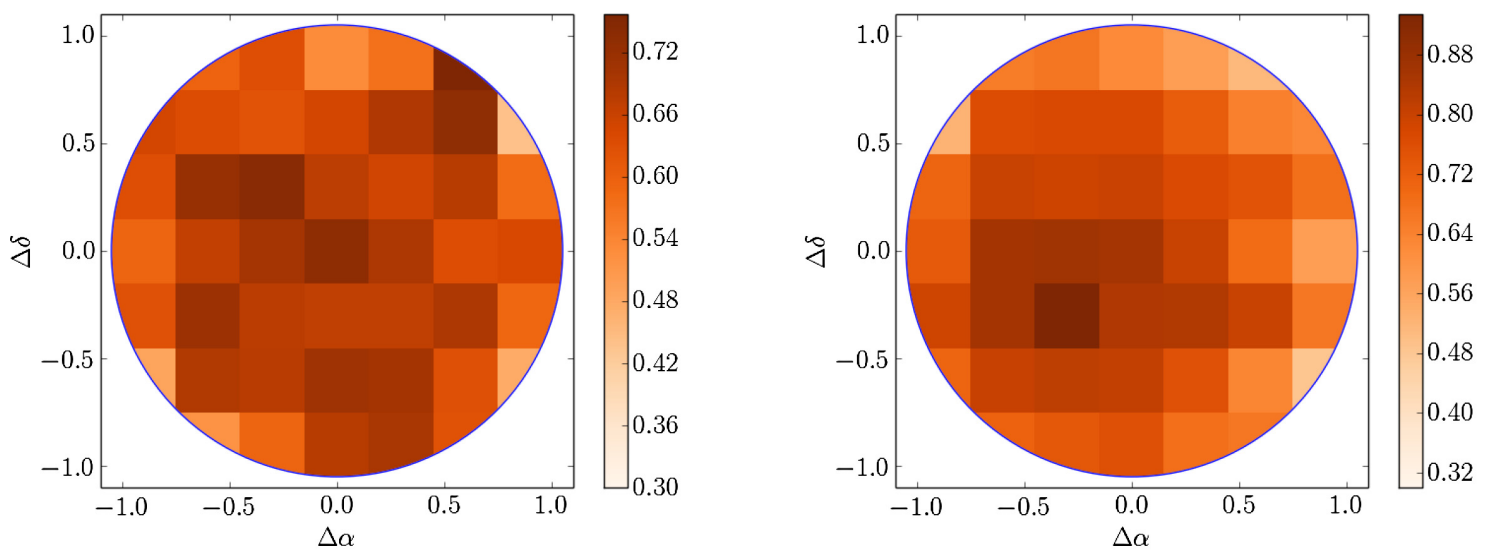

Figure 13. Redshift completeness in bins of $0.3 \mathrm{deg}$ by $0.3 \mathrm{deg}$ for SN hosts (left) and LRGs (right). The blue circle represents the 2.1-deg diameter of the 2dF field of view. The decreasing redshift completeness with distance from the centre of $2 \mathrm{dF}$ is a consequence of poorer image quality at larger radii. The presence of a gradient in the right plot and not the left one is surprising.
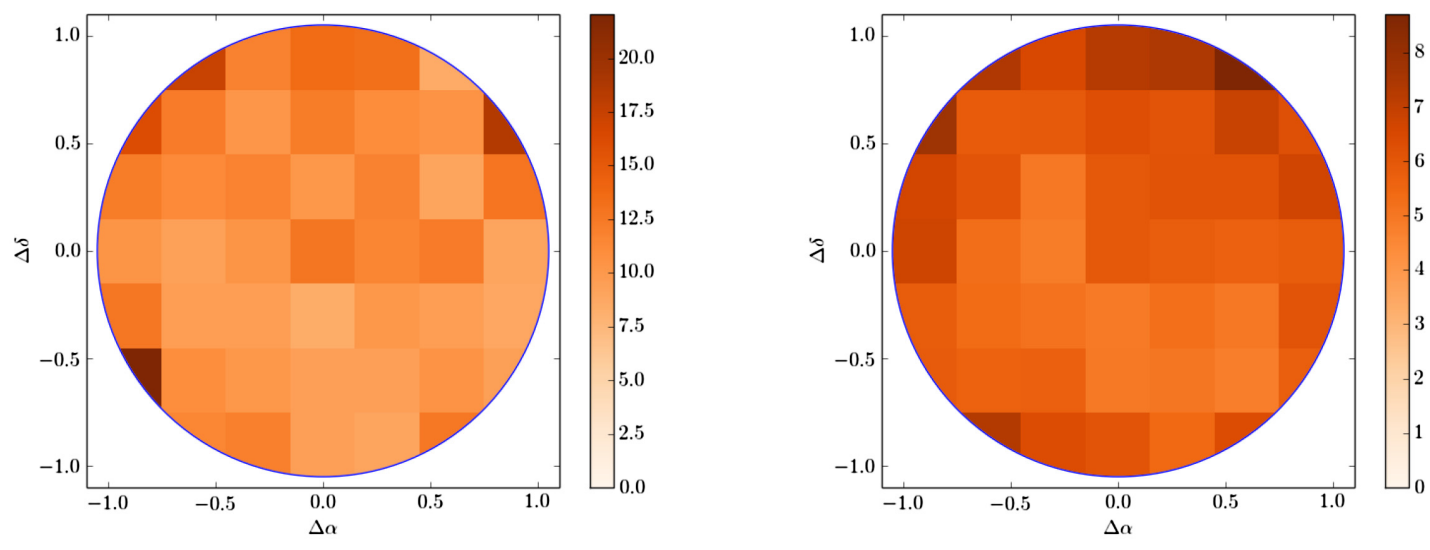

Figure 14. The average number of exposures, in bins of $0.3 \mathrm{deg}$ by $0.3 \mathrm{deg}$, required for a successful redshift measurement for SN hosts (left) and LRGs (right). The blue circle represents the 2.1-deg diameter of the $2 \mathrm{dF}$ field of view. Overall, as one approaches edges of the $2 \mathrm{dF}$ field of view, it takes more exposures to secure a redshift. The gradient from lower left to upper right seen in Fig. 13 is also seen here and is also stronger for the LRGs.

In OzDES-DR2, we release 375000 spectra of almost 39000 objects, of which three quarters have a redshift with a quality flag of 3 or greater. We release all spectra, including the spectra of objects that do not have a redshift. These data can used to help secure redshifts for these objects if they are observed in the future with the AAT or other spectroscopic facilities. A full description of the data release is provided in Appendix B.

\section{AGN RM}

Second to SN hosts, AGN were the next most frequently observed target type. As shown in Fig. 2, about a quarter of the fibre hours available to OzDES were allocated to the AGN RM programme. The first year of the OzDES RM programme was used to define the sample that was followed in the subsequent 5 years. Details of the selection are provided in King et al. (2015). In brief, AGN were selected on the basis of the quality of the spectra that were taken in $\mathrm{Y} 1$, on the presence of emission lines, while avoiding AGN that occasionally fell in chip gaps, maximizing the number of AGN with two reverberating spectral lines in our wavelength range, and ensuring that the AGN selected sampled the redshift interval $0<$ $z<4$. Most AGN that pass these cuts have $r_{\mathrm{AB}}<21$.2. From an initial sample of 3331 AGN, 771 AGN were followed during the next 5 years.
Apart from active transients, AGN in the OzDES RM sample have the highest priority, so the percentage of AGN that were allocated a fibre in any given observation was near to 100 per cent. However, not all fields could be observed during every OzDES run, because of a combination of the finite duration of the observing runs, time lost to poor observing conditions, and the differing priority of the 10 DES deep fields. Considering the latter and the bar chart shown in Fig. 3, we expect that the AGN in the two E-region fields and the $\mathrm{X} 3$ and $\mathrm{C} 3$ fields will have the greatest number of observations, both in terms of the raw number of times they were observed, and the number of runs in which they were observed.

In Fig. 15, we show the distribution of the number of runs in which AGN were observed. Two plots are shown. In the lower plot, all observations are included, regardless of the observing conditions or the quality of the data. In the upper plot, only data that had a relative zero-point greater than -2 (see Fig. 4 ) and a quality control (QC) flag of 'ok' are included (see Appendix B for a discussion on the QC flag). The vertical solid line shows the number of runs on which OzDES observations were scheduled during the 6 years that OzDES ran. The two runs from the OzDES pre-survey are excluded, since the AGN RM pre-sample had not been defined at that time. This solid line represents an upper limit to the number of epochs. The median values for the four regions are shown in Table 4. The median values for the $\mathrm{X}$ and $\mathrm{C}$ regions include $\mathrm{X} 3$ and 

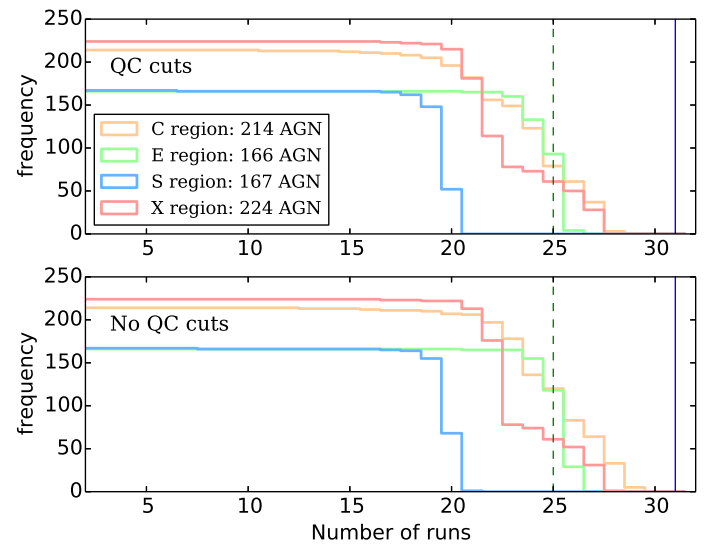

Figure 15. Reverse cumulative histograms of the number of independent spectroscopic epochs in the AGN reverberation mapping programme, plotted without restriction on data quality (lower histogram) and excluding poor quality data (upper histogram). See the text for details. The vertical line corresponds to the number of runs on which some OzDES data were taken during the 6 years that OzDES ran. It corresponds to the maximum possible number of epochs. The prediction on the number of recovered lags made in King et al. (2015) was based on obtaining 25 epochs, which is shown as the dotted line.

Table 4. Median number of AGN epochs.

\begin{tabular}{lc}
\hline Fields & Epochs \\
\hline C1, C2, and C3 & 23 \\
E1 and E2 & 24 \\
S1 and S2 & 18 \\
X1, X2, and X3 & 21 \\
C3 & 25 \\
X3 & 25 \\
\hline
\end{tabular}

C3, which were the highest priority fields, so we list the median values for the $\mathrm{C} 3$ and $\mathrm{X} 3$ separately as well. For the E-region fields and $\mathrm{X} 3$ and $\mathrm{C} 3$, the median is close to the 25 epochs that were used in the simulations of the OzDES RM programme in King et al. (2015).

With this number of epochs, simulations in King et al. (2015) predict that one should recover lags for 35-45 percent of the AGN depending on the accuracy with which one can calibrate the line flux. The simulations used a 10-per cent accuracy for the line measurements. Hoormann et al. (2019) demonstrate that we are able to achieve this level of accuracy, so we anticipate that we will recover this fraction of lags in the two E fields and the $\mathrm{C} 3$ and X3 fields, and a lower fraction in the other six fields. The first two lags, using the C IV line and based on a subset of the OzDES data, were published in Hoormann et al. (2019).

\section{SN HOST GALAXIES: A CASE STUDY}

As illustrated in Fig. 2, slightly more than one third of all the fibre hours allocated to targets during the 6 years of OzDES were allocated to SN hosts. Unlike other targets, SN hosts were observed until a secure redshift (see Section 4) was obtained. SN hosts also extend to fainter magnitudes than most targets. Both of these factors meant that the integration time for some hosts is very long, as long as 2 days in a number of cases.

We use SN hosts to examine how well the measured noise changes with time, and explore the implications of deselecting SN hosts once they reach a $Q=3$ quality redshift and before they reach the more secure $Q=4$ quality redshift.

\subsection{Dependence on the measured noise with integration time}

Ch17 showed that the noise in the spectra evolves with increasing exposure time, $t$, according to expectations. The OzDES pipeline averages spectra from individual exposures rather than summing them, so the measured noise decreases as $\sqrt{t}$, or with the square root of the number of exposures if the exposure time is constant. Nearly all OzDES exposures were taken with an integration time of $40 \mathrm{~min}$. We repeat the Ch17 analysis, which was done with just 3 years of OzDES data. With 6 years of data, there are more objects in total and there are more objects with longer integration times.

We do apply some qualitative cuts to the data chosen for this analysis. We exclude spectra that (i) were taken in poor conditions (defined here as exposures that have a relative zero-point of less than -2; see Fig. 4), (ii) did not pass QC, and (iii) were taken during Y1 and SV. During Y1 and SV, we do not have reliable zero-points, and did not take dome flats, so the quality of the processed data is not as good as it was in later years.

With these cuts, we then select SN hosts that have at least 12 exposures. This will tend to select SN hosts that are fainter than average, as redshifts for bright hosts would have typically been obtained with fewer exposures and would have been deselected from the target pool before 12 exposures had been taken. As the selected objects are faint, the signal-to-noise ratio is quite small, so spectral features do not contribute to the noise.

We then select a wavelength region over which to measure the noise. We start off with a region that is relatively free of night sky lines. The region we select starts at $6610 \AA$ and ends at $6750 \AA$. We then examine how our results change if we choose a region that contains bright night sky lines.

We then randomize the order in which exposures were taken. The ordering is different for each object. For a given object, we take the first exposure and compute the clipped RMS over the wavelength region noted above. In computing the clipped RMS, we reject the five highest and five lowest values. Over the wavelength region that we sample, there are about 135 pixels, so the clipping removes less than 10 percent of the data. The clipping allows us to exclude residuals from cosmic rays that were not perfectly removed during the processing of the data and mitigates the effect of strong spectral features, such as bright emission lines from the object. We experimented with the normalized median absolute deviation instead of the clipped RMS and obtained qualitatively similar results. All subsequent measurements of the clipped RMS are scaled by the clipped RMS that is computed in this first exposure.

We then add data from subsequent exposures in the randomized sequence, noting that we average the spectra, rather than summing them, and recompute the clipped RMS. This is done for every SN host. The results are shown in Fig. 16.

There is considerable scatter from object to object. This is caused by data of poorer than average quality entering the average at different stages of the sequence. For example, a sequence of exposures in which the first exposure is noisy will result in a much larger than anticipated drop in the relative clipped RMS when data of better quality are included. Applying stronger quality cuts reduces the scatter. To get an idea of the general trend, we take the median of all objects. These are the red circles in Fig. 16. 


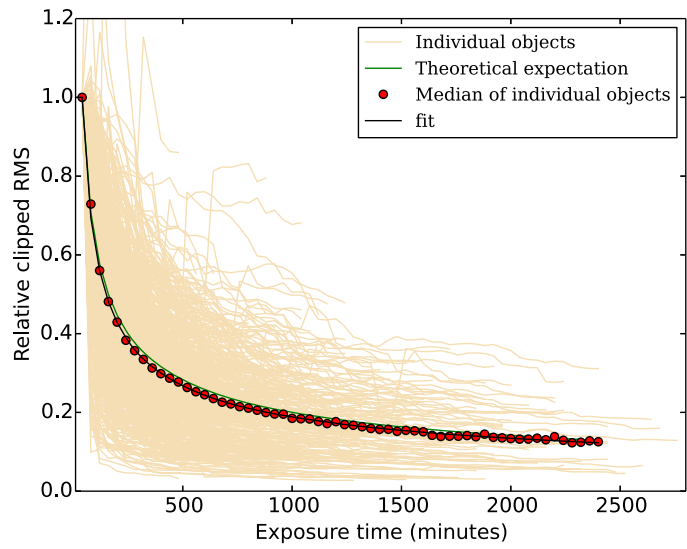

Figure 16. The scaled clipped RMS of 639 individual objects (in light yellow) from the three $\mathrm{C}$ fields plotted against exposure time. The median of these curves is plotted as the solid red circles. The theoretical expectation is the green curve and the best fit is the black curve. Note how closely the two match. The clipped RMS is computed over the region 6610-6750.

We fit the function

$\frac{n^{\alpha}+\beta}{1+\beta}$

to the red points. The function equals 1 when $n=1$, and allows for a noise floor. The theoretical expectation for Poisson noise is $\alpha=-0.5$ and $\beta=0.0$. This is represented with the green curve. The best fit to equation (1) is the black curve. The best fit has $\alpha=$ $-0.54 \pm 0.01$ and $\beta=0.02 \pm 0.01$. The best fit is close to theoretical expectation for Poisson noise, and the floor is low. We find there is no reason to stop observing targets over the range of exposure times explored here. We will explore this point further in the next section.

There are limitations to our approach. Equation (1) may not be the best functional form, and we have not allowed for the hard limits imposed by theory (i.e. $\beta \geq 0$ and $\alpha \geq-0.5$ ) when fitting equation (1). Indeed, we find $\alpha<-0.5$. It is unclear what causes this.

We varied our selection cuts and repeated the analysis for the other fields to see how robust our results were. We do see some variability in the results, the origins of which are unclear. For the permutations we tried, we found $-0.55<\alpha<-0.5$ and $0<\beta<$ 0.05 , i.e. a slightly steeper dependence than expected from theory and weak evidence for a noise floor. The floor is still less than the contribution from Poisson noise even after 1 day of exposure. At 1.5 days (which corresponds to 54 40-min exposures), $54^{-0.5} \sim 0.14$.

We also varied the wavelength region over which the statistics were computed, using both a region free of bright night sky lines (6610-6750 $\AA$ ) and a region dominated by them (7700-8000 $)$. In both cases, a dependence close to $1 / \sqrt{n}$ was observed.

\subsection{Deselecting targets}

It is clear that, for the range of exposure times explored in OzDES (up to 2 days of integration), the signal-to-noise continues to improve as expected, i.e. with the square root of the exposure time. If OzDES were to continue in the future, then exposure times could grow into many days. A number of questions then arise. How long should one spend on a target before deselecting it for an another one if one is unable to get a redshift, and is there a way of knowing if some targets are more likely to result in a redshift than others? Any decision to deselect one source in preference for another needs careful consideration, as such a decision will invariably lead to biases that need to be modelled.

During Y6, when 71 per cent of the available fibre time was spent targeting $\mathrm{SN}$ hosts, there were more $\mathrm{SN}$ hosts without quality 4 redshifts than $2 \mathrm{dF}$ fibres, especially for $\mathrm{C} 3$ and $\mathrm{X} 3$, the two deepest DES fields. If there were many more fibres than the 392 available with $2 \mathrm{dF}$, and $\mathrm{SN}$ hosts were the only targets, then the answer would be to continue observing these targets.

For instruments like 4MOST, which has 4 times as many fibres as $2 \mathrm{dF}$, one could observe more targets longer without running out of fibres. Of course, 4MOST could choose to push to fainter limits than OzDES did (most OzDES hosts are brighter than $\left.r_{\mathrm{AB}}=24\right)$, in which case it too would have to make a choice about what to keep and what to drop in favour of something that has a higher chance of success.

Ultimately, this decision has to be based on the questions one is trying to address. An SN with a noisy light curve at $z \sim 1$ may not be as valuable as two $\mathrm{SNe}$ with high-quality light curves at a redshift of a half. We do not address this question in this paper, but leave it to when the DES 5-years photometrically selected sample is defined.

For $\mathrm{SN}$ hosts, there is an additional consideration. $\mathrm{SN}$ hosts are deselected once they get a $Q=4$ quality redshift. Some $\mathrm{SN}$ hosts obtain a $Q=3$ quality redshift relatively quickly, but they fail to reach the $Q=4$ quality redshift that is necessary for deselection. This raises an interesting question. How long should one continue observing such targets once the $Q=3$ quality redshift is obtained?

We examine the number of exposures $\mathrm{SN}$ hosts remain at $Q=$ 3 once that quality is obtained. We divide these objects into two. Those that eventually obtain a $Q=4$ quality redshift and those that do not.

Almost 85 percent of objects that move from $Q=3$ to 4 do so within 10 exposures of obtaining $Q=3$. If one were to deselect targets that fail to reach $Q=4$ within 10 exposures of obtaining $Q=$ 3 , then one would free up 6.6 per cent of the fibre hours, which could then be used on other sources, at the cost of obtaining 6.2 per cent fewer $Q=4$ quality redshifts.

In OzDES, we did not deselect $\mathrm{SN}$ hosts before they reached $Q=$ 4 , no matter how many exposures were being taken. We are now in position to explore how the choice of deselecting $\mathrm{SN}$ hosts biases the fitted cosmological parameters. This will be reported in a future paper.

About $35 \mathrm{SN}$ hosts fainter than $r=23$ fail to obtain a $Q=4$ quality redshift once a $Q=3$ quality redshift was obtained, even after continuing to integrate for 30 or more exposures (equivalent to $20 \mathrm{~h}$ of integration). We examined each of these sources, and found that more than half of them show a single bright line, which is assumed to be the [O II] doublet. These objects reach the $Q=3$ level relatively quickly. The redshift of these sources is around one, meaning that other potential emission lines were outside the spectral range of AAOmega. During run041, we used a redder setting for AAOmega, which allowed us to pick up the [O III] doublet in some cases. Most of the remaining cases consist of sources with weak features, typically a combination of weak [O II], [O III], and $\mathrm{H} \beta$, that only start to become apparent after many hours of integration, but they are not yet strong enough for a $Q=4$ quality redshift to be assigned, even after an additional $20 \mathrm{~h}$ of additional integration.

\section{IMPLICATIONS FOR FUTURE SURVEYS}

By the early 2020s, LSST will have started surveying the Southern sky and several new multi-object spectroscopic facilities fed by 
Table 5. A comparison between TiDES/4MOST and OzDES/AAOmega.

\begin{tabular}{lcc}
\hline Metric & OzDES/AAT & TiDES/4MOST \\
\hline \multicolumn{2}{c}{ Telescope, Spectrograph, and Positioner } \\
M1 diameter (m) & 3.9 & 4.2 \\
Field of view (sq. deg.) & 3.46 (circle) & 4.2 (hexagon) \\
Fibre diameter (arcsec) & $2.0-2.1$ & 1.45 \\
Number of fibres & 392 & $1624^{a}$ \\
Config. time (min) & $40^{b}$ & 2 \\
Spectral resolution & $1400-1700$ & 6500 \\
Wavelength range (A) & $3700-8800$ & $3700-9500$ \\
Smallest sep. (arcsec) & 30 & 15 \\
& \multicolumn{3}{c}{} \\
Survey region ${ }^{c, d}$ & DES deep fields & LSST DDF and \\
& 1.5 & $+5^{\circ}<\delta<-70^{\circ}$ \\
Median seeing (arcsec) & 67 per cent & 0.8 \\
Usable clear fraction & $5^{g}$ & 90 per cent \\
Duration (years) & 5000 & 5 \\
Transient redshifts & 771 & 50000 \\
AGN monitored & 1450 & 700 \\
Live transients & 193000 & $30000^{e}$ \\
Fibre hours & 1 & 250000 \\
Freq. (per lunation) & 5 months & $2^{f}$ \\
Observing season & 9 months $s^{f}$ \\
\hline
\end{tabular}

Notes. ${ }^{a}$ Fibres feeding the two low-resolution spectrographs.

${ }^{b}$ Done in parallel while observing with the opposing plate.

${ }^{c}$ The 10 DES deep fields cover 27 sq. deg.

${ }^{d}$ Deep Drilling Fields.

${ }^{e}$ Mostly from the TiDES wide survey.

${ }^{f}$ In the deep drilling fields.

$g$ The SN programme in DES and OzDES lasted five seasons.

rapidly configurable fibre positioners covering wide fields of view will be operational. Of the planned surveys using these new facilities, the Time-Domain Extragalactic Survey (TiDES; Swann et al. 2019), using 4MOST (de Jong et al. 2019) in combination with LSST, is the one that most closely matches the OzDES concept. A comparison of the OzDES and TiDES surveys is shown in Table 5. The survey characteristics of TiDES listed in Table 5 are subject to change as the survey strategy is still evolving.

On practically every metric, TiDES will be superior to OzDES. It will observe at least 10 times more live transients, mostly in the wide survey, it will measure up to 10 times more host galaxy redshifts, and it will monitor AGN at twice the frequency that OzDES did and with shorter seasonal gaps. TiDES will also observe transients over the majority of the southern sky, something that OzDES did not do.

Although the science goals and strategy of TiDES are heavily based upon that of OzDES, practically there are some differences. The first difference between OzDES and TiDES is that TiDES will not drive the pointings of 4MOST. Wherever 4MOST is scheduled to point there will be previously discovered transient targets, a majority of these targets anticipated to be discovered by LSST. If these transients are still bright enough to obtain a spectrum for spectral typing, TiDES will attempt do so. Otherwise, TiDES will target the host galaxy of the transient to obtain a spectroscopic redshift for type Ia $\mathrm{SN}$ cosmology.

The second difference between OzDES and TiDES is that TiDES will have two components, a wide-field component and a deep field component. The wide field follow-up will cover the majority of the southern sky, where low-redshift transients and host galaxies will be targeted with a maximum exposure time of $2 \mathrm{~h}$. The second component to TiDES will be spectroscopic follow-up in the deep fields of 4MOST, where higher redshift live transients will be observed, the AGN RM will take place, and where one can repeatedly target $\mathrm{SN}$ host galaxies to build up redshifts on the faintest of targets.

The 4MOST Deep fields currently correspond to four pointings, each covering $4.2 \mathrm{sq}$. deg and contained within the LSST deep drilling fields (Guiglion et al. 2019). The LSST data will provide the high-precision photometry and high-cadence light curves required for sub-percent precision cosmology. As three of the DES deep fields (ECDFS, XMM-LSS, and ELAIS S1) are also covered by the 4MOST and LSST DDFs, this offers the exciting possibility of continued monitoring a subsample of the AGN observed by OzDES over a 15-year baseline, if the time gap between OzDES and TiDES can be covered. The higher cadence and longer observing season will also mean that TiDES will be more sensitive to AGN with shorter lags and less sensitive to seasonal gaps. Therefore, TiDES will obtain a higher fraction of lags compared to OzDES.

With more than 4 times the number of fibres, and a field of view that is only slightly larger than $2 \mathrm{dF}$, TiDES on 4MOST will be able to observe $\mathrm{SN}$ hosts in the deep fields longer without running out of fibres. Given the higher throughput, broader spectral coverage, and higher spectral resolution of 4MOST compared to AAOmega, and the better observing conditions on Cerro Paranal compared to Siding Spring, TiDES will be able to obtain host galaxy redshifts more quickly and to a fainter magnitude limit than OzDES

\section{SUMMARY}

Over six consecutive observing seasons, starting in 2013, OzDES obtained over 375000 spectra of almost 39000 sources in the 10 DES deep fields. These spectra were obtained with the $2 \mathrm{dF}$ fibre positioner and AAOmega spectrograph on the AAT. Over that time, each DES SN field was observed between 18 and 25 times.

In these fields, OzDES has obtained redshifts for almost 7000 galaxies that hosted a transient, and classified almost 270 transients. The strategy of targeting each field repeatedly has enabled redshifts to be obtained for sources as faint as $r_{\mathrm{AB}} \sim 24$. We show that one is not limited by a noise floor, even after 2 days of exposure, and that one can reach high spectroscopic completeness at these faint limits.

The strategy of targeting each field repeatedly has allowed OzDES to monitor 771 AGN. These data will be used to measure the lag between the continuum from the accretion disc and the broad lines from the broad line emission region. We anticipate that we will recover lags and derive black hole masses for approximately 30 per cent of the AGN we monitored.

The OzDES observing strategy allowed a number of science programmes to run in parallel. Just over 60 per cent of the 192000 fibre hours were allocated to monitoring AGN and obtaining redshifts for galaxies that hosted a transient. The remaining 40 per cent was used to obtain redshifts for a wide variety of targets, such as radio galaxies, cluster galaxies, and galaxies to train photometric redshift algorithms.

OzDES can be used as a template for future surveys that use the next generation of wide-field multi-object fibre-fed spectroscopic facilities to target and monitor sources in the LSST deep drilling fields. These new facilities will have up to an order of magnitude more fibres than $2 \mathrm{dF}$, and they will enable one to follow a larger number of fainter sources more frequently and for longer. 


\section{ACKNOWLEDGEMENTS}

Parts of this research were supported by the Australian Research Council under grants DP160100930, FL180100168, and FT140101270.

Based in part on data acquired at the AAT, under program $\mathrm{A} / 2013 \mathrm{~B} / 012$. We acknowledge the traditional owners of the land on which the AAT stands, the Gamilaraay people, and pay our respects to elders past, present and emerging.

We acknowledge the support of James Tocknell and Simon O'Toole from Data Central in helping us prepare OzDES-DR2.

LG was funded by the European Union's Horizon 2020 research and innovation program under the Marie Skłodowska-Curie grant agreement no. 839090 .

FHP was supported by an Australian Government Research Training Program Scholarship.

Funding for the DES Projects has been provided by the U.S. Department of Energy, the U.S. National Science Foundation, the Ministry of Science and Education of Spain, the Science and Technology Facilities Council of the United Kingdom, the Higher Education Funding Council for England, the National Center for Supercomputing Applications at the University of Illinois at UrbanaChampaign, the Kavli Institute of Cosmological Physics at the University of Chicago, the Center for Cosmology and Astro-Particle Physics at the Ohio State University, the Mitchell Institute for Fundamental Physics and Astronomy at Texas A\&M University, Financiadora de Estudos e Projetos, Fundação Carlos Chagas Filho de Amparo à Pesquisa do Estado do Rio de Janeiro, Conselho Nacional de Desenvolvimento Científico e Tecnológico and the Ministério da Ciência, Tecnologia e Inovação, the Deutsche Forschungsgemeinschaft, and the Collaborating Institutions in the DES.

The Collaborating Institutions are Argonne National Laboratory, the University of California at Santa Cruz, the University of Cambridge, Centro de Investigaciones Energéticas, Medioambientales y Tecnológicas-Madrid, the University of Chicago, University College London, the DES-Brazil Consortium, the University of Edinburgh, the Eidgenössische Technische Hochschule (ETH) Zürich, Fermi National Accelerator Laboratory, the University of Illinois at Urbana-Champaign, the Institut de Ciències de l'Espai (IEEC/CSIC), the Institut de Física d'Altes Energies, Lawrence Berkeley National Laboratory, the Ludwig-Maximilians Universität München and the associated Excellence Cluster Universe, the University of Michigan, the National Optical Astronomy Observatory, the University of Nottingham, The Ohio State University, the University of Pennsylvania, the University of Portsmouth, SLAC National Accelerator Laboratory, Stanford University, the University of Sussex, Texas A\&M University, and the OzDES Membership Consortium.

Based in part on observations at Cerro Tololo Inter-American Observatory, National Optical Astronomy Observatory, which is operated by the Association of Universities for Research in Astronomy (AURA) under a cooperative agreement with the National Science Foundation.

The DES data management system is supported by the National Science Foundation under Grant Numbers AST-1138766 and AST-1536171. The DES participants from Spanish institutions are partially supported by MINECO under grants AYA201571825, ESP2015-66861, FPA2015-68048, SEV-2016-0588, SEV2016-0597, and MDM-2015-0509, some of which include ERDF funds from the European Union. IFAE is partially funded by the CERCA program of the Generalitat de Catalunya. Research leading to these results has received funding from the European Research Council under the European Union's Seventh Framework Program
(FP7/2007-2013) including ERC grant agreements 240672, 291329, and 306478. We acknowledge support from the Brazilian Instituto Nacional de Ciência e Tecnologia (INCT) e-Universe (CNPq grant 465376/2014-2).

This manuscript has been authored by Fermi Research Alliance, LLC under Contract No. DE-AC02-07CH11359 with the U.S. Department of Energy, Office of Science, and Office of High Energy Physics. The United States Government retains and the publisher, by accepting the article for publication, acknowledges that the United States Government retains a non-exclusive, paid-up, irrevocable, world-wide license to publish or reproduce the published form of this manuscript, or allow others to do so, for United States Government purposes.

We are grateful for the extraordinary contributions of our CTIO colleagues and the DECam Construction, Commissioning and Science Verification teams in achieving the excellent instrument and telescope conditions that have made this work possible. The success of this project also relies critically on the expertise and dedication of the DES Data Management group.

\section{REFERENCES}

Angus C. R. et al., 2019, MNRAS, 487, 2215

Astier P. et al., 2006, A\&A, 447, 31

Bernstein J. P. et al., 2012, ApJ, 753, 152

Betoule M. et al., 2014, A\&A, 568, A22

Bonnett C. et al., 2016, Phys. Rev. D, 94, 042005

Calcino J., Davis T., 2017, J. Cosmol. Astropart. Phys., 2017, 038

Childress M. J. et al., 2017, MNRAS, 472, 273

Croom S., Saunders W., Heald R., 2004, Anglo-Aust. Obs. Epping Newsl., 106, 12

D'Andrea C. B. et al., 2018, preprint (arXiv:1811.09565)

Dark Energy Survey, 2016, MNRAS, 460, 1270

Dark Energy Survey, 2019a, Phys. Rev. Lett., 122, 171301

Dark Energy Survey, 2019b, ApJ, 872, L30

Davies L. J. M. et al., 2018, MNRAS, 480, 768

Dawson K. S. et al., 2009, AJ, 138, 1271

de Jong R. S. et al., 2019, The Messenger, 175, 3

DESI Collaboration, 2016, preprint (arXiv:1611.00036)

Diehl H. T. et al., 2018, in Alison B. P., Robert L. S., Chris R. B., eds, Proc. SPIE Conf. Ser. Vol. 10704, Observatory Operations: Strategies, Processes, and Systems VII. SPIE, Bellingham, p. 107040D

Flaugher B. et al., 2015, AJ, 150, 150

Franzen T. M. O. et al., 2015, MNRAS, 453, 4020

Gschwend J. et al., 2018, Astron. Comput., 25, 58

Guiglion G. et al., 2019, The Messenger, 175, 17

Hinton S. R., Davis T. M., Lidman C., Glazebrook K., Lewis G. F., 2016, Astron. Comput., 15, 61

Hoormann J. K. et al., 2019, MNRAS, 487, 3650

Jacobs C. et al., 2019, MNRAS, 484, 5330

Johnson A. et al., 2017, MNRAS, 465, 4118

Khain T. et al., 2018, AJ, 156, 273

King A. L. et al., 2015, MNRAS, 453, 1701

Lawrence J. et al., 2018, in Christopher J. E., Luc S., Hideki T., eds, Proc. SPIE Conf. Ser. Vol. 10702, Ground-Based and Airborne Instrumentation for Astronomy VII. SPIE, Bellingham, p. 10702A6

Li T. S. et al., 2019, MNRAS, 490, 3508

LSST Science Collaboration, 2017, preprint (arXiv:1708.04058)

Marshall J. et al., 2019, ApJ, 882, 177

Mudd D. et al., 2018, ApJ, 862, 123

Nord B. et al., 2016, ApJ, 827, 51

Palmese A. et al., 2017, ApJ, 849, L34

Pierre M. et al., 2016, A\&A, 592, A1

Prat J. et al., 2018, Phys. Rev. D, 98, 042005

Pursiainen M. et al., 2018, MNRAS, 481, 894

Rozo E. et al., 2016, MNRAS, 461, 1431 
Sánchez C. et al., 2014, MNRAS, 445, 1482

Smith M. et al., 2018, ApJ, 854, 37

Swann E. et al., 2019, The Messenger, 175, 58

Tamura N. et al., 2018, in Christopher J. E., Luc S., Hideki T., eds, Proc. SPIE Conf. Ser. Vol. 10702, Ground-Based and Airborne Instrumentation for Astronomy VII. SPIE, Bellingham, p. 107021C

The MSE Science Team et al., 2019, preprint (arXiv:1904.04907)

Vargas-Magana M., Brooks D. D., Levi M. M., Tarle G. G., 2019, preprint (arXiv:1901.01581)

Webb T. M. A. et al., 2015, ApJ, 814, 96

Wilson G. et al., 2009, ApJ, 698, 1943

Yasuda N., et al., 2019, PASJ, 71, 74

Yu Z. et al., ApJS, 2020, 246, 16

Yuan F. et al., 2015, MNRAS, 452, 3047

\section{SUPPORTING INFORMATION}

Supplementary data are available at MNRAS online.

Appendix A. Observing logs for Y4, Y5, and Y6.

Appendix B. The second OzDES Data release-OzDES-DR2.

Appendix C. Source types for the MaxVis field.

Please note: Oxford University Press is not responsible for the content or functionality of any supporting materials supplied by the authors. Any queries (other than missing material) should be directed to the corresponding author for the article.

${ }^{1}$ The Research School of Astronomy and Astrophysics, Australian National University, Stromlo, ACT 2601, Australia

${ }^{2}$ School of Mathematics and Physics, University of Queensland, Brisbane, QLD 4072, Australia

${ }^{3}$ The Observatories of the Carnegie Institution for Science, 813 Santa Barbara St, Pasadena, CA 91101, USA

${ }^{4}$ Centro de Investigaciones Energéticas, Medioambientales y Tecnológicas (CIEMAT), Av. Complutense, 40, E-28040 Madrid, Spain

${ }^{5}$ School of Natural Sciences, College of Sciences and Engineering, University of Tasmania, Private Bag 37, Hobart, TAS 7001, Australia

${ }^{6}$ Department of Physics and Astronomy, University of Pennsylvania, Philadelphia, PA 19104, USA

${ }^{7}$ INAF, Astrophysical Observatory of Turin, I-10025 Pino Torinese, Turin, Italy

${ }^{8}$ School of Physics and Astronomy, University of Southampton, Southampton SO17 1BJ, UK

${ }^{9}$ Santa Cruz Institute for Particle Physics, Santa Cruz, CA 95064, USA

${ }^{10}$ PITT PACC, Department of Physics and Astronomy, University of Pittsburgh, Pittsburgh, PA 15260, USA

${ }^{11}$ Centre for Astrophysics and Supercomputing, Swinburne University of Technology, Melbourne, VIC 3122, Australia

${ }^{12}$ Department of Astronomy and Astrophysics, University of Chicago, Chicago, IL 60637, USA

${ }^{13}$ Kavli Institute for Cosmological Physics, University of Chicago, Chicago, IL 60637, USA

${ }^{14}$ Lawrence Berkeley National Laboratory, 1 Cyclotron Road, Berkeley, CA 94720, USA

${ }^{15}$ School of Physics, University of Melbourne, Parkville, VIC 3010, Australia ${ }^{16}$ Department of Physics, University of Michigan, Ann Arbor, MI 48109, USA

${ }^{17}$ Australian Astronomical Optics, Macquarie University, North Ryde, NSW 2113, Australia

${ }^{18}$ Lowell Observatory, 1400 Mars Hill Rd, Flagstaff, AZ 86001, USA

${ }^{19}$ Centre for Extragalactic Astronomy, Department of Physics, Durham University, Durham DH1 3LE, UK

${ }^{20}$ Institute for Computational Cosmology, Durham University, South Road, Durham DH1 3LE, UK
${ }^{21}$ Sydney Institute for Astronomy, School of Physics, A28, The University of Sydney, Sydney, NSW 2006, Australia

${ }^{22}$ Institute of Cosmology and Gravitation, University of Portsmouth, Portsmouth PO1 3FX, UK

${ }^{23}$ Center for Cosmology and Astro-Particle Physics, The Ohio State University, Columbus, OH 43210, USA

${ }^{24}$ Department of Astronomy, The Ohio State University, Columbus, OH 43210, USA

${ }^{25}$ Université Clermont Auvergne, CNRS/IN2P3, LPC, F-63000 ClermontFerrand, France

${ }^{26}$ School of Science, UNSW Canberra, Australian Defence Force Academy, Canberra, ACT 2612, Australia

${ }^{27}$ Korea Astronomy and Space Science Institute, 776 Daedeokdae-ro, Yuseong-gu, 34055 Daejeon, Republic of Korea

${ }^{28}$ Centre for Translational Data Science, University of Sydney, Darlington, NSW 2008, Australia

${ }^{29}$ Department of Physics, Duke University Durham, Science Dr, Durham, NC 27708, USA

${ }^{30}$ Cerro Tololo Inter-American Observatory, National Optical Astronomy Observatory, Casilla 603, La Serena, Chile

${ }^{31}$ Departamento de Física Matemática, Instituto de Física, Universidade de São Paulo, CP 66318, São Paulo, SP 05314-970, Brazil

${ }^{32}$ Laboratório Interinstitucional de e-Astronomia - LIneA, Rua Gal. José Cristino 77, Rio de Janeiro, RJ 20921-400, Brazil

${ }^{33}$ Fermi National Accelerator Laboratory, PO Box 500, Batavia, IL 60510, USA

${ }^{34}$ Instituto de Fisica Teorica UAM/CSIC, Universidad Autonoma de Madrid, C/ Nicolás Cabrera, 13-15, E-28049 Madrid, Spain

${ }^{35}$ CNRS, UMR 7095, Institut d'Astrophysique de Paris, 98bis Boulevard Arago, F-75014 Paris, France

${ }^{36}$ Sorbonne Universités, UPMC Univ Paris 06, UMR 7095, Institut d'Astrophysique de Paris, 12 Rue Cuvier, F-75014 Paris, France

${ }^{37}$ Department of Physics and Astronomy, Pevensey Building, University of Sussex, Brighton BN1 9QH, UK

${ }^{38}$ Department of Physics and Astronomy, University College London, Gower Street, London WC1E 6BT, UK

${ }^{39}$ Department of Astronomy, University of Illinois at Urbana-Champaign, 1002 W. Green Street, Urbana, IL 61801, USA

${ }^{40}$ National Center for Supercomputing Applications, 1205 West Clark St, Urbana, IL 61801, USA

${ }^{41}$ Institut de Física d'Altes Energies (IFAE), The Barcelona Institute of Science and Technology, Campus UAB, E-08193 Bellaterra (Barcelona), Spain

${ }^{42}$ Institut d'Estudis Espacials de Catalunya (IEEC), Gran Capità, 2-4, Edifici Nexus, despatx 201, E-08034 Barcelona, Spain

${ }^{43}$ Institute of Space Sciences (ICE, CSIC), Campus UAB, Carrer de Can Magrans, s/n, E-08193 Barcelona, Spain

${ }^{44}$ INAF-Osservatorio Astronomico di Trieste, via G. B. Tiepolo 11, I-34143

Trieste, Italy

${ }^{45}$ Institute for Fundamental Physics of the Universe, Via Beirut 2, I-34014 Trieste, Italy

${ }^{46}$ Observatório Nacional, Rua Gal. José Cristino 77, Rio de Janeiro, RJ 20921-400, Brazil

${ }^{47}$ Department of Astronomy/Steward Observatory, University of Arizona, 933 North Cherry Avenue, Tucson, AZ 85721-0065, USA

${ }^{48}$ Jet Propulsion Laboratory, California Institute of Technology, 4800 Oak Grove Dr., Pasadena, CA 91109, USA

${ }^{49}$ Department of Physics, Stanford University, 382 Via Pueblo Mall, Stanford, CA 94305, USA

${ }^{50}$ Kavli Institute for Particle Astrophysics and Cosmology, Stanford University, PO Box 2450, Stanford, CA 94305, USA

${ }^{51}$ SLAC National Accelerator Laboratory, Menlo Park, CA 94025, USA

${ }^{52}$ Département de Physique Théorique and Center for Astroparticle Physics, Université de Genève, 24 quai Ernest Ansermet, CH-1211 Geneva, Switzerland

${ }^{53}$ Department of Physics, ETH Zurich, Wolfgang-Pauli-Strasse 16, CH-8093 Zurich, Switzerland 
${ }^{54}$ Department of Physics, The Ohio State University, Columbus, $\mathrm{OH} 43210$, USA

${ }^{55}$ Center for Astrophysics | Harvard \& Smithsonian, 60 Garden Street, Cambridge, MA 02138, USA

${ }^{56}$ Department of Astrophysical Sciences, Princeton University, Peyton Hall, Princeton, NJ 08544, USA

${ }^{57}$ Observatories of the Carnegie Institution for Science, 813 Santa Barbara St, Pasadena, CA 91101, USA

${ }^{58}$ George P. and Cynthia Woods Mitchell Institute for Fundamental Physics and Astronomy, and Department of Physics and Astronomy, Texas A\&M University, College Station, TX 77843, USA

${ }^{59}$ Institució Catalana de Recerca i Estudis Avançats, Passeig de Lluís Companys, 23, E-08010 Barcelona, Spain
${ }^{60}$ Instituto de Física, UFRGS, Caixa Postal 15051, Porto Alegre, RS 91501970, Brazil

${ }^{61}$ Computer Science and Mathematics Division, Oak Ridge National Laboratory, Oak Ridge, TN 37831, USA

${ }^{62}$ Max Planck Institute for Extraterrestrial Physics, Giessenbachstrasse, D-85748 Garching, Germany

${ }^{63}$ Universitäts-Sternwarte, Fakultät für Physik, Ludwig-Maximilians Universität München, Scheinerstr 1, D-81679 München, Germany

This paper has been typeset from a $\mathrm{T}_{\mathrm{E}} \mathrm{X} / \mathrm{LAT}_{\mathrm{E}} \mathrm{X}$ file prepared by the author. 